\title{
Complete and consistent chiral transport from Wigner function formalism
}

\author{
Anping Huang, ${ }^{1}$ Shuzhe Shi, ${ }^{2}$ Yin Jiang, ${ }^{3}$ Jinfeng Liao, ${ }^{2, *}$ and Pengfei Zhuang ${ }^{1, \dagger}$ \\ ${ }^{1}$ Physics Department, Tsinghua University, Beijing 100084, China \\ ${ }^{2}$ Physics Department and Center for Exploration of Energy and Matter, Indiana University, \\ 2401 N Milo B. Sampson Lane, Bloomington, Indiana 47408, USA \\ ${ }^{3}$ School of Physics and Nuclear Energy Engineering, Beihang University, Beijing 100191, China
}

(Received 2 February 2018; published 15 August 2018)

\begin{abstract}
Recently, there has been significant interest in understanding the macroscopic quantum transport in a many-body system of chiral fermions. A natural framework for describing such a system that is generally out of equilibrium is the transport equation for its phase space distribution function. In this paper, we obtain a complete solution of the covariant chiral transport for massless fermions, by starting from the general Wigner function formalism and carrying out a complete and consistent semiclassical expansion up to $\hat{\mathbf{O}}(\hbar)$ order. In particular, we clarify certain subtle and confusing issues surrounding the Lorentz noninvariance and frame dependence associated with the three-dimensional chiral kinetic theory. We prove that such frame dependence is uniquely and completely fixed by an unambiguous definition of the $\hat{\mathbf{O}}(\hbar)$ correction to the distribution function in each reference frame.
\end{abstract}

DOI: 10.1103/PhysRevD.98.036010

\section{INTRODUCTION}

The many-body physics of massless fermions has attracted significant interest in a wide range of communities, from condensed matter physics to high-energy heavy ion collisions. In particular, the microscopic quantum anomaly of such chiral fermions can induce highly nontrivial macroscopic transport phenomena, such as the notable example of the chiral magnetic effect (CME) [1-4] as well as the chiral vortical effect [5-7]. These effects have been extensively studied using various manybody theoretical tools [5,8-19]. Enthusiastic efforts have also been made to experimentally measure such anomalous chiral transport effects, both in the so-called Dirac or Weyl semimetals and in the so-called quark-gluon plasma created via heavy ion collisions. For reviews on recent developments, see, e.g., Refs. [20-24].

An important aspect of the many-body theory for anomalous chiral transport is to describe the out-ofequilibrium situation. The natural framework is the kinetic theory based on transport equations for the phase space distribution function of such a system. Different from usual classical kinetic theory [25], a proper description of the

\footnotetext{
*liaoji@indiana.edu

†huangpf@mail.tsinghua.edu.cn
}

Published by the American Physical Society under the terms of the Creative Commons Attribution 4.0 International license. Further distribution of this work must maintain attribution to the author(s) and the published article's title, journal citation, and DOI. Funded by SCOAP ${ }^{3}$. chiral fermions must account for intrinsic quantum and relativistic effects. A lot of progress has been achieved lately to develop such a chiral kinetic theory; see, e.g., Refs. [26-40]. There also exist a lot of phenomenological interest and attempts to study anomalous chiral transport in the out-of-equilibrium setting [41-50]. The transport theory of chiral fermions, however, bears unusual subtlety and poses a number of challenges, particularly related to Lorentz invariance and frame dependence. A resolution was developed in the three-dimensional (3D) formulation of chiral kinetic theory [29,30,35], but the origin of such issues remains cloudy. It is highly desirable to develop a transport theory of chiral fermions in a completely covariant fashion and to identify the precise reason of these complications.

A natural approach is to derive the quantum transport equation for chiral fermions in the well-known Wigner function formalism by a systematic semiclassical expansion in terms of $\hbar$ [51-56]. We shall adopt this approach in the present paper. We will systematically derive the chiral transport equations for a general out-of-equilibrium system of collisionless massless fermions, under external electromagnetic fields that are generally space-time dependent. The starting point is the Wigner function and the kinetic equation for Winger function and its 16 components, such as the vector $\mathscr{V}_{\mu}$, axial vector $\mathscr{A}_{\mu}$, scalar $\mathscr{F}$, pseudoscalar $\mathscr{P}$, and antisymmetry tensor $\mathscr{L}^{\mu \nu}$. These 16 components would be decoupled for the chiral fermion system. We will focus on the set of equations for vector $\mathscr{V}^{\mu}$ and axial vector $\mathscr{A}^{\mu}$ components. By carrying out the semiclassical expansion for all the operators and functions, one can then derive 
a covariant set of chiral transport equations to $\hbar$ order. In particular, this detailed derivation will allow a clear understanding, within a totally covariant framework, of the origin for the rather confusing Lorentz-invariance and framedependence issues as well as the emergence of the Berry phase, Berry curvature and anomalous terms in the 3D formulation of chiral kinetic theory. In fact, we will prove that such frame dependence is uniquely and completely fixed by an unambiguous definition of the $\hat{\mathbf{O}}(\hbar)$ correction to the distribution function in each reference frame.

The paper is organized as follows. In Sec. II, we briefly review the Wigner function formalism and the kinetic equations for the 16 components of Wigner function. In Sec. III, these equations are decoupled and decompose to two sets of equations for the massless case, and we focus on the semiclassical expansion for the chiral currents. With the obtained constraint equations, we construct the most general solutions and discuss the frame-dependence issue. In Sec. IV, we present the covariant chiral transport equations as well as their 3D formulation. Finally, we conclude the paper in In Sec. V. An Appendix is also included to particularly prove in great technical details the completeness and uniqueness of the found $\hat{\mathbf{O}}(\hbar)$ solution to the constraint equations, which is crucial for understanding the frame-dependence issue.

\section{QUANTUM KINETIC EQUATIONS IN THE WIGNER FUNCTION FORMALISM}

The bridge connecting quantum field theory to relativistic kinetic theory is the Wigner function [55,57]. For the Dirac field $\psi$ with charge $Q$, the general gauge-invariant Wigner operator is defined as

$\hat{W}_{\alpha \beta}(x, p)=\int \frac{d^{4} y}{(2 \pi)^{4}} e^{-\frac{i}{\hbar} p \cdot y} \bar{\psi}_{\beta}\left(x_{+}\right) U\left(x_{+}, x_{-}\right) \psi_{\alpha}\left(x_{-}\right)$,

where $\alpha$ and $\beta$ are spinor indices. Also, the gauge link $U$ between $x_{ \pm}=x \pm y / 2$ is introduced to ensure the gauge invariance of the Wigner operator. It is defined as

$$
U\left(x_{+}, x_{-}\right)=\mathcal{P} e^{-\frac{i Q}{h} y^{\mu} \int_{0}^{1} d s A_{\mu}\left(x-\frac{y}{2}+s y\right)},
$$

where the path-ordering operator $\mathcal{P}$ can be dropped for Abelian $A^{\mu}$ fields. In this work, we keep the Planck constant $\hbar$ in various places to show the quantum effect explicitly.

Then, one can construct the Winger function, as the expectation value of the Wigner operator

$$
W_{\alpha \beta}(x, p)=\left\langle\hat{W}_{\alpha \beta}(x, p)\right\rangle,
$$

where $\langle\cdots\rangle$ means the expectation over a given quantum state, or the average over an ensemble of quantum states.
In this work, we consider a collisionless system in a background electromagnetic field $A^{\mu}$. In this case, the Wigner function satisfies the quantum kinetic equation [55]

$$
(K-m) W(x, p)=0,
$$

where $K=\gamma^{\mu} \mathbf{K}_{\mu}, \mathbf{K}_{\mu}=\pi_{\mu}+\frac{1}{2} i \hbar \nabla_{\mu}$, and

$$
\begin{gathered}
\pi^{\mu}=p^{\mu}-\frac{1}{2} Q \hbar j_{1}\left(\frac{1}{2} \hbar \Delta\right) F^{\mu \nu} \partial_{\nu}^{p}, \\
\nabla^{\mu}=\partial^{\mu}-Q j_{0}\left(\frac{1}{2} \hbar \Delta\right) F^{\mu \nu} \partial_{\nu}^{p} .
\end{gathered}
$$

Note that in the triangle operator $\Delta=\partial_{x} \cdot \partial_{p}, \partial_{x}$ acts only on electromagnetic tensor $F_{\mu \nu}=\partial^{\mu} A^{\nu}-\partial^{\nu} A^{\mu}$, while $\partial_{p}$ acts only on $W(x, p)$. In addition, $j_{0}(x)=x^{-1} \sin (x)$ and $j_{1}(x)=x^{-2} \sin (x)-x^{-1} \cos (x)$ are the spherical Bessel functions that are generated by the y integrations. In general combining with the Maxwell equation, the quantum kinetic equation of the Wigner function (4) is equivalent to the QED field theory.

To connect Eq. (4) with kinetic theory, one needs to obtain explicitly the equations of all elements of the Wigner function, which is a $4 \times 4$ matrix. To do that, one can decompose the $W(x, p)$ in terms of the 16 generators of the Clifford algebra, choosing the convention basis as follows:

$$
\begin{aligned}
& \Gamma^{a}=I, \gamma^{\mu}, i \gamma^{5}, \gamma^{\mu} \gamma^{5}, \sigma^{\mu \nu}, \\
& \Gamma_{a}=I, \gamma_{\mu},-i \gamma_{5}, \gamma_{5} \gamma_{\mu}, \sigma_{\mu \nu} .
\end{aligned}
$$

In this basis, the Wigner function is expanded as

$W=\frac{1}{4}\left(\mathscr{F}+i \gamma^{5} \mathscr{P}+\gamma^{\mu} \mathscr{V}_{\mu}+\gamma^{\mu} \gamma^{5} \mathscr{A}_{\mu}+\frac{1}{2} \sigma^{\mu \nu} \mathscr{L}_{\mu \nu}\right)$,

where these 16 components are given by

$$
\begin{aligned}
\mathscr{F}(x, p) & =\operatorname{tr} W(x, p), \\
\mathscr{P}(x, p) & =-i \operatorname{tr}\left[\gamma_{5} W(x, p)\right], \\
\mathscr{V}_{\mu}(x, p) & =\operatorname{tr}\left[\gamma_{\mu} W(x, p)\right], \\
\mathscr{A}_{\mu}(x, p) & =\operatorname{tr}\left[\gamma_{5} \gamma_{\mu} W(x, p)\right], \\
\mathscr{L}_{\mu \nu}(x, p) & =\operatorname{tr}\left[\sigma_{\mu \nu} W(x, p)\right]=-\mathscr{L}_{\nu \mu}(x, p) .
\end{aligned}
$$

Noting that the Wigner function satisfies Hermiticity relations $W^{\dagger}(x, p)=\gamma^{0} W(x, p) \gamma^{0}$ in the same way as the $\Gamma_{a}$ 's $\left(\Gamma_{a}^{\dagger}=\gamma^{0} \Gamma_{a} \gamma^{0}\right)$, all these 16 components are real, and they behave as the scalar, pseudoscalar, vector, axial vector, and antisymmetric tensor, respectively, under Lorentz transformation. Each of these 16 components is connected with a corresponding physical quantity $[51,58]$. Explicitly speaking, the vector $\mathcal{V}_{\mu}$ and axial vector $\mathcal{A}_{\mu}$ can be used to construct the current density $J^{\mu}$, axial current density $J_{5}^{\mu}$, and energy-momentum tensor $T^{\mu \nu}$, 


$$
\begin{aligned}
J^{\mu}(x) & =\left\langle\bar{\psi}(x) \gamma^{\mu} \psi(x)\right\rangle=\int d^{4} p \operatorname{tr}\left(\gamma^{\mu} W(x, p)\right)=\int d^{4} p \mathcal{V}^{\mu}(x, p), \\
J_{5}^{\mu}(x) & =\left\langle\bar{\psi}(x) \gamma^{\mu} \gamma^{5} \psi(x)\right\rangle=-\int d^{4} p \operatorname{tr}\left(\gamma^{5} \gamma^{\mu} W(x, p)\right)=-\int d^{4} p \mathscr{A}^{\mu}(x, p), \\
T^{\mu \nu}(x) & =\frac{-i}{2}\left\langle\bar{\psi}(x)\left[\gamma^{\mu} D^{\nu+}-\gamma^{\mu} D^{\nu}\right] \psi(x)\right\rangle=\int d^{4} p p^{\nu} \operatorname{tr}\left(\gamma^{\mu} W(x, p)\right)=\int d^{4} p p^{\nu} \mathscr{V}^{\mu}(x, p),
\end{aligned}
$$

with the covariant derivative $D^{\mu}=\partial^{\mu}+i Q A^{\mu}$ (where $Q$ is the charge of fermion and $A^{\mu}$ is the vector potential). Note that the above definition of the energy-momentum tensor is the so-called canonical definition, which in principle should be further symmetrized in the Lorentz indices $\mu$ and $\nu$ as is typically done in quantum field theory textbooks.

Now, we can derive the kinetic equations for these 16 coefficients explicitly. Substituting the decomposed Wigner function (8) into Eq. (4), one obtains

$$
\begin{aligned}
0= & \left(\gamma^{\mu} \mathbf{K}_{\mu} \mathscr{F}+i \gamma^{\mu} \gamma^{5} \mathbf{K}_{\mu} \mathscr{P}+\gamma^{\mu} \gamma^{\nu} \mathbf{K}_{\mu} \mathscr{V}_{\nu}+\gamma^{\mu} \gamma^{\nu} \gamma^{5} \mathbf{K}_{\mu} \mathscr{A}_{\nu}+\frac{1}{2} \gamma^{\mu} \sigma^{\nu \sigma} \mathbf{K}_{\mu} \mathscr{L}_{\nu \sigma}\right) \\
& -m\left(\mathscr{F}+i \gamma^{5} \mathscr{P}+\gamma^{\mu} \mathscr{V}_{\mu}+\gamma^{\mu} \gamma^{5} \mathscr{A}_{\mu}+\frac{1}{2} \sigma^{\mu \nu} \mathscr{L}_{\mu \nu}\right) .
\end{aligned}
$$

Next, we will use the properties of the $\gamma$ matrices [with the metric convention $g^{\mu \nu}=\operatorname{diag}(1,-1,-1,-1)$ and the Levi-Cività antisymmetric tensor $\left.\epsilon^{0123}=-\epsilon_{0123}=1\right]$,

$$
\begin{aligned}
\left\{\gamma^{\mu}, \gamma^{5}\right\} & =0, \quad\left\{\gamma^{\mu}, \gamma^{\nu}\right\}=2 g^{\mu \nu}, \quad \gamma^{\mu} \gamma^{\nu}=g^{\mu \nu}-i \sigma^{\mu \nu}, \\
\sigma^{\mu \nu} \gamma^{5} & =\frac{i}{2} \epsilon^{\mu \nu \sigma \rho} \sigma_{\sigma \rho}, \quad \gamma^{\sigma} \sigma^{\mu \nu}=g^{\sigma \mu} \gamma^{\nu}-g^{\sigma \nu} \gamma^{\mu}+i \epsilon^{\mu \nu \sigma \rho} \gamma_{\rho} \gamma^{5},
\end{aligned}
$$

to cast terms with multiple $\gamma$ matrices into the $\Gamma_{a}$ basis:

$$
\begin{aligned}
\gamma^{\mu} \gamma^{\nu} \mathbf{K}_{\mu} \mathcal{V}_{\nu} & =\mathbf{K}_{\mu} \mathscr{V}^{\mu}-\frac{i}{2} \sigma^{\mu \nu}\left(\mathbf{K}_{\mu} \mathscr{V}_{\nu}-\mathbf{K}_{\nu} \mathscr{V}_{\mu}\right), \\
\gamma^{\mu} \gamma^{\nu} \gamma^{5} \mathbf{K}_{\mu} \mathscr{A}_{\nu} & =-i\left(i \gamma^{5}\right) \mathbf{K}_{\mu} \mathscr{A}^{\mu}+\frac{1}{2} \epsilon_{\mu \nu \sigma \rho} \sigma^{\mu \nu} \mathbf{K}^{\sigma} \mathscr{A}^{\rho}, \\
\gamma^{\mu} \sigma^{\nu \sigma} \mathbf{K}_{\mu} \mathscr{L}_{\nu \sigma} & =-2 i \gamma^{\mu} \mathbf{K}^{\nu} \mathscr{L}_{\mu \nu}+\epsilon_{\mu \nu \sigma \rho} \gamma^{\mu} \gamma^{5} \mathbf{K}^{\nu} \mathscr{L}^{\sigma \rho} .
\end{aligned}
$$

These relations allow us to simplify Eq. (11) as

$$
\begin{aligned}
& I\left(\mathbf{K}_{\mu} \mathscr{V}^{\mu}-m \mathscr{F}\right)+i \gamma^{5}\left(-i \mathbf{K}_{\mu} \mathscr{A}^{\nu}-m \mathscr{P}\right)+\gamma^{\mu}\left(\mathbf{K}_{\mu} \mathscr{F}-i \mathbf{K}^{\nu} \mathscr{L}_{\mu \nu}-m \mathscr{V}_{\mu}\right) \\
& \quad+\gamma^{\mu} \gamma^{5}\left(i \mathbf{K}_{\mu} \mathscr{P}+\frac{1}{2} \epsilon_{\mu \nu \sigma \rho} \mathbf{K}^{\nu} \mathscr{L}^{\sigma \rho}-m \mathscr{A}_{\mu}\right)+\frac{1}{2} \sigma^{\mu \nu}\left(-i\left(\mathbf{K}_{\mu} \mathcal{V}_{\nu}-\mathbf{K}_{\nu} \mathscr{V}_{\mu}\right)+\epsilon_{\mu \nu \sigma \rho} \mathbf{K}^{\sigma} \mathscr{A}^{\rho}-m \mathscr{L}_{\mu \nu}\right)=0
\end{aligned}
$$

From the orthogonality of the $\left\{\Gamma_{a}\right\}$ basis, i.e., $\operatorname{tr}\left(\Gamma_{a} \Gamma_{b}\right)=4 \delta_{a b}$, one can prove that all "elements" of the above "matrix" should be zero, i.e.,

$$
\begin{gathered}
0=\mathbf{K}_{\mu} \mathscr{V}^{\mu}-m \mathscr{F}, \\
0=i \mathbf{K}_{\mu} \mathscr{A}^{\mu}+m \mathscr{P}, \\
0=\mathbf{K}_{\mu} \mathscr{F}-i \mathbf{K}^{\nu} \mathscr{L}_{\mu \nu}-m \mathscr{V}_{\mu}, \\
0=i \mathbf{K}_{\mu} \mathscr{P}+\frac{1}{2} \epsilon_{\mu \nu \sigma \rho} \mathbf{K}^{\nu} \mathscr{L}^{\sigma \rho}-m \mathscr{A}_{\mu}, \\
0=i\left(\mathbf{K}_{\mu} \mathscr{V}_{\nu}-\mathbf{K}_{\nu} \mathscr{V}_{\mu}\right)-\epsilon_{\mu \nu \sigma \rho} \mathbf{K}^{\sigma} \mathscr{A}^{\rho}+m \mathscr{L}_{\mu \nu} .
\end{gathered}
$$

Furthermore, as $\mathbf{K}^{\mu}=\pi^{\mu}+\frac{1}{2} i \hbar \nabla^{\mu}$ is complex while all components of the Wigner function are real, one could further separate the above equations with the real and imaginary parts. The real parts give

$$
\begin{gathered}
m \mathscr{F}=\pi^{\mu} \mathscr{V}_{\mu}, \\
2 m \mathscr{P}=\hbar \nabla^{\mu} \mathscr{A}_{\mu}, \\
m \mathscr{V}_{\mu}=\pi_{\mu} \mathscr{F}+\frac{1}{2} \hbar \nabla^{\nu} \mathscr{L}_{\mu \nu}, \\
2 m \mathscr{A}_{\mu}=-\hbar \nabla_{\mu} \mathscr{P}+\epsilon_{\mu \nu \sigma \rho} \pi^{\nu} \mathscr{L}^{\sigma \rho}, \\
m \mathscr{L}_{\mu \nu}=\frac{1}{2} \hbar\left(\nabla_{\mu} \mathscr{V}_{\nu}-\nabla_{\nu} \mathscr{V}_{\mu}\right)+\epsilon_{\mu \nu \sigma \rho} \pi^{\sigma} \mathscr{A}^{\rho},
\end{gathered}
$$


while the imaginary parts lead to

$$
\begin{gathered}
\hbar \nabla^{\mu} \mathscr{V}_{\mu}=0, \\
\pi^{\mu} \mathscr{A}_{\mu}=0, \\
\frac{1}{2} \hbar \nabla_{\mu} \mathscr{F}=\pi^{\nu} \mathscr{L}_{\mu \nu}, \\
\pi_{\mu} \mathscr{P}=-\frac{1}{4} \hbar \epsilon_{\mu \nu \sigma \rho} \nabla^{\nu} \mathscr{L}^{\sigma \rho}, \\
\pi_{\mu} \mathscr{V}_{\nu}-\pi_{\nu} \mathscr{V}_{\mu}=\frac{1}{2} \hbar \epsilon_{\mu \nu \sigma \rho} \nabla^{\sigma} \mathscr{A}^{\rho} .
\end{gathered}
$$

The above results are the complete quantum kinetic equations [51-55], as shown in Eqs. (18)-(27), in terms of the 16 components of the Wigner function that are coupled with each other. In the next section, we will focus on the massless case to further simplify the kinetic equations.

\section{CHIRAL TRANSPORT EQUATIONS AND THE GENERAL SOLUTIONS}

In this section, we consider a system of chiral fermions with $m=0$. In this case, the quantum kinetic equations in Eqs. (18)-(27) get partially decoupled. One can see explicitly that they are separated into two groups: a set of equations describing the evolution of scalar $\mathscr{F}$, pseudoscalar $\mathscr{P}$, and antisymmetry tensor $\mathscr{L}^{\mu \nu}$ components,

$$
\begin{aligned}
\pi_{\mu} \mathscr{F}+\frac{1}{2} \hbar \nabla^{\nu} \mathscr{L}_{\mu \nu} & =0, \\
\frac{1}{2} \hbar \nabla_{\mu} \mathscr{F}-\pi^{\nu} \mathscr{L}_{\mu \nu} & =0, \\
-\hbar \nabla_{\mu} \mathscr{P}+\epsilon_{\mu \nu \rho \sigma} \pi^{\nu} \mathscr{L}^{\rho \sigma} & =0, \\
\pi_{\mu} \mathscr{P}+\frac{1}{4} \hbar \epsilon_{\mu \nu \rho \sigma} \nabla^{\nu} \mathscr{L}^{\rho \sigma} & =0,
\end{aligned}
$$

and another set for vector $\mathscr{V}_{\mu}$ and axial vector $\mathscr{A}_{\mu}$ components,

$$
\begin{aligned}
\pi^{\mu} \mathscr{V}_{\mu} & =0, \quad \pi^{\mu} \mathscr{A}_{\mu}=0, \\
\hbar \nabla^{\mu} \mathscr{V}_{\mu} & =0, \quad \hbar \nabla^{\mu} \mathscr{A}_{\mu}=0, \\
\hbar \epsilon_{\mu \nu \rho \sigma} \nabla^{\rho} \mathscr{V}^{\sigma} & =2\left(\pi_{\mu} \mathscr{A}_{\nu}-\pi_{\nu} \mathscr{A}_{\mu}\right), \\
\hbar \epsilon_{\mu \nu \rho \sigma} \nabla^{\rho} \mathscr{A}^{\sigma} & =2\left(\pi_{\mu} \mathscr{V}_{\nu}-\pi_{\nu} \mathscr{V}_{\mu}\right) .
\end{aligned}
$$

Noting the specific patterns of vector (scalar) and axialvector (pseudoscalar) terms, one could further simplify the above two sets of equations by introducing the "chiral basis" $[34,54]$ via

$$
\begin{aligned}
\mathscr{T}_{\chi} & =\frac{1}{2}(\mathscr{F}+\chi \mathscr{P}), \\
\mathcal{S}_{\chi}^{\mu \nu} & =\frac{1}{2}\left(\mathscr{L}^{\mu \nu}+\chi \frac{1}{2} \epsilon^{\mu \nu \sigma \rho} \mathscr{L}_{\sigma \rho}\right), \\
\mathscr{J}_{\chi}^{\mu} & =\frac{1}{2}\left(\mathscr{V}^{\mu}-\chi \mathscr{A}^{\mu}\right),
\end{aligned}
$$

where $\chi= \pm 1$ corresponds to the chirality of massless fermion. In such a chiral basis, Eq. (28) can be further decomposed, in which the right-handed (RH) and lefthanded (LH) components get decoupled:

$$
\begin{aligned}
& \pi_{\mu} \mathscr{T}_{\chi}+\frac{1}{2} \hbar \nabla^{\nu} \mathcal{S}_{\mu \nu}^{\chi}=0, \\
& \pi_{\mu} \mathcal{\delta}_{\chi}^{\mu \nu}+\frac{1}{2} \hbar \nabla^{\nu} \mathscr{T}_{\chi}=0 .
\end{aligned}
$$

Similarly, Eq. (29) can be recast into RH and LH sectors:

$$
\begin{gathered}
\hbar \epsilon_{\mu \nu \rho \sigma} \nabla^{\rho} \mathscr{F}_{\chi}^{\sigma}=-2 \chi\left(\pi_{\mu} \mathscr{F}_{\nu}^{\chi}-\pi_{\nu} \mathscr{F}_{\mu}^{\chi}\right), \\
\pi^{\mu} \mathscr{F}_{\mu}^{\chi}=0, \\
\nabla^{\mu} \mathscr{F}_{\mu}^{\chi}=0 .
\end{gathered}
$$

The decoupling of the RH and LH components in these equations reflects a basic property of massless fermions: for the massless Dirac fermions, the RH and LH sectors can be completely separated in the Lagrangian.

As the main purpose of this paper is to study the chiral transport effects, we will focus on the equations for the chiral components $\mathscr{F}_{\chi}^{\mu}$, namely, Eqs. (33)-(35) in the following. We note in passing that the chiral components $\mathscr{F}_{\chi}^{\mu}$ can be directly related to the physical chiral currents:

$$
J_{\chi}^{\mu}=\left\langle\bar{\psi}_{\chi} \gamma^{\mu} \gamma^{5} \psi_{\chi}\right\rangle=\int d^{4} p \mathscr{F}_{\chi}^{\mu}=\frac{1}{2}\left(J^{\mu}+\chi J_{5}^{\mu}\right) .
$$

Here, $\psi_{\chi}=P_{\chi} \psi$ and $\bar{\psi}_{\chi}=\bar{\psi} P_{-\chi}$, with $P_{\chi}=\left(1+\chi \gamma^{5}\right) / 2$ being the chirality projection operators.

\section{A. Semiclassical expansion}

We now derive the chiral kinetic equation, by starting from Eqs. (33)-(35) and utilizing the semiclassical expansion method [55]. To do this, one needs to expand both operators and Wigner function components in the evolution equations order by order in terms of $\hbar$. First of all, let us expand the operators $\pi^{\mu}$ and $\nabla^{\mu}$ in powers of $\hbar$, by using the Taylor expansion of the spherical Bessel function $j_{0}$ and $j_{1}$ in terms of $\frac{1}{2} \hbar \Delta$, with $j_{0}(x)=1-x^{2} / 6+\mathbf{O}\left(x^{4}\right)$ and $j_{1}(x)=x / 3-x^{3} / 30+\mathbf{O}\left(x^{5}\right)$ : 


$$
\begin{aligned}
\pi^{\mu} & =p^{\mu}-\frac{1}{2} Q \hbar j_{1}\left(\frac{1}{2} \hbar \Delta\right) F^{\mu \nu} \partial_{\nu}^{p}=p^{\mu}-\frac{1}{12} Q \hbar^{2} \Delta F^{\mu \nu} \partial_{\nu}^{p}+\mathbf{O}\left(\hbar^{4}\right), \\
\nabla^{\mu} & =\partial^{\mu}-Q j_{0}\left(\frac{1}{2} \hbar \Delta\right) F^{\mu \nu} \partial_{\nu}^{p}=\partial^{\mu}-Q F^{\mu \nu} \partial_{\nu}^{p}+\frac{1}{24} Q \hbar^{2} \Delta^{2} F^{\mu \nu} \partial_{\nu}^{p}+\mathbf{O}\left(\hbar^{4}\right) .
\end{aligned}
$$

The truncation of this expansion series would be justified when $\frac{1}{2} \hbar\left|\partial_{x} F^{\mu \nu} \cdot \partial_{p} W(x, p)\right| \ll\left|F^{\mu \nu} W(x, p)\right|$. In other words, the electromagnetic field $F^{\mu \nu}$ and Wigner function $W(x, p)$ should vary smoothly enough in coordinate space $x$ and momentum space $p$, respectively [55,59]. It should be emphasized that, starting from here through the rest of this paper, we will use the notation $\nabla^{\mu}$ to represent its zeroth-order truncation, i.e., $\nabla^{\mu} \rightarrow \partial^{\mu}-Q F^{\mu \nu} \partial_{\nu}^{p}$.

We next write down an expansion of $\mathscr{f}_{\chi}^{\mu}$ also in powers of $\hbar$, i.e.,

$$
\mathscr{J}_{\chi, \mu}=\mathscr{F}_{\chi, \mu}^{(0)}+\hbar \mathscr{F}_{\chi, \mu}^{(1)}+\hbar^{2} \mathscr{F}_{\chi, \mu}^{(2)}+\mathbf{O}\left(\hbar^{3}\right) .
$$

By substituting the operators in Eq. (37) and chiral component in Eq. (38) into Eqs. (33)-(35), one obtains

$$
\begin{gathered}
0=\left[p_{\mu} \mathscr{F}_{\chi, \nu}^{(0)}-p_{\nu} \mathscr{J}_{\chi, \mu}^{(0)}\right] \\
+\hbar\left[\epsilon_{\mu \nu \rho \sigma} \nabla^{\rho} \mathscr{F}_{\chi}^{(0) \sigma}+2 \chi\left(p_{\mu} \mathscr{F}_{\chi, \nu}^{(1)}-p_{\nu} \mathcal{F}_{\chi, \mu}^{(1)}\right)\right]+\mathbf{O}\left(\hbar^{2}\right) \\
0=\left[p^{\mu} \mathscr{F}_{\chi, \mu}^{(0)}\right]+\hbar\left[p^{\mu} \mathscr{F}_{\chi, \mu}^{(1)}\right]+\mathbf{O}\left(\hbar^{2}\right) \\
0=\left[\nabla^{\mu} \mathscr{F}_{\chi, \mu}^{(0)}\right]+\hbar\left[\nabla^{\mu} \mathscr{J}_{\chi, \mu}^{(1)}\right]+\mathbf{O}\left(\hbar^{2}\right)
\end{gathered}
$$

Just as with the strategy in perturbation theory, one can then match the terms in the above equations at each given order of $\hbar$ and obtain an infinite series of equations order by order. In this paper, we will only deal with the two leading orders, i.e., the order $\hbar^{0}$ equations and the order $\hbar^{1}$ equations.

Let us first examine the zeroth-order equations:

$$
\begin{gathered}
0=p_{\mu} \mathscr{J}_{\chi, \nu}^{(0)}-p_{\nu} \mathscr{F}_{\chi, \mu}^{(0)}, \\
0=p^{\mu} \mathscr{F}_{\chi, \mu}^{(0)}, \\
0=\nabla^{\mu} \mathscr{F}_{\chi, \mu}^{(0)} .
\end{gathered}
$$

Equations (42) and (43) are the constraint conditions for the current $\mathscr{F}_{\chi, \mu}^{(0)}$; the former requires that $\mathscr{F}_{\chi, \mu}^{(0)}$ must be parallel to $p_{\mu}$, i.e., $\mathscr{F}_{\chi, \mu}^{(0)}=p_{\mu} S(x, p)$, where $S$ is a certain arbitrary scalar function, and the latter further demands that $p^{2} S(x, p)=0$. These conditions uniquely fix the general form of the zeroth-order current to be

$$
\mathscr{J}_{\mu, \chi}^{(0)}=p_{\mu} f_{\chi}^{(0)} \delta\left(p^{2}\right)
$$

together with the classical on-shell condition as reflected in the delta function. Apparently, $f_{\chi}^{(0)}$ is the classical phasespace distribution function, which can be further decomposed as

$$
f_{\chi}^{(0)}(x, p)=\sum_{\epsilon= \pm 1} \theta\left(\epsilon p^{0}\right) f_{\chi}^{(0) \epsilon}(x, \epsilon p)
$$

where $\epsilon= \pm 1$ corresponds to particle with positive/ negative energy.

Finally, by substituting Eq. (45) into the evolution equation (44), one obtains the zeroth-order transport equation,

$\delta\left(p^{2}\right) p^{\mu} \nabla_{\mu} f_{\chi}^{(0)}=\delta\left(p^{2}\right) p^{\mu}\left(\partial_{\mu}-Q F_{\mu \nu} \partial_{p}^{\nu}\right) f_{\chi}^{(0)}=0$,

which is the classical covariant Vlasov equation. Note that in deriving the above result we have used the relations concerning derivative terms, $\nabla^{\mu} p_{\mu}=-Q F_{\mu}^{\mu}=0$ as well as $p_{\mu} f_{\chi}^{(0)} \nabla^{\mu} \delta\left(p^{2}\right)=-2 Q p_{\mu} F^{\mu \nu} p_{\nu} f_{\chi}^{(0)} \delta^{\prime}\left(p^{2}\right)=0$, both vanishing by virtue of the antisymmetric nature of the electromagnetic field tensor.

\section{B. $\hbar$-order constraint equations and general solutions}

We now move on to examine the first-order equations, as follows:

$$
\begin{gathered}
0=\epsilon_{\mu \nu \rho \sigma} \nabla^{\rho} \mathscr{F}_{\chi}^{(0) \sigma}+2 \chi\left(p_{\mu} \mathscr{F}_{\chi, \nu}^{(1)}-p_{\nu} \mathscr{F}_{\chi, \mu}^{(1)}\right), \\
0=p^{\mu} \mathscr{F}_{\chi, \mu}^{(1)}, \\
0=\nabla^{\mu} \mathscr{F}_{\chi, \mu}^{(1)} .
\end{gathered}
$$

Here, Eq. (48) gives the connection between the zeroth and first orders of $\mathscr{J}_{\mu}$. Noting that

$$
\begin{aligned}
\nabla^{\rho} \mathscr{F}_{\chi}^{(0) \sigma}= & -Q F^{\rho \sigma} f_{\chi}^{(0)} \delta\left(p^{2}\right)+p^{\sigma}\left(\nabla^{\rho} f_{\chi}^{(0)}\right) \delta\left(p^{2}\right) \\
& -2 Q F^{\rho \lambda} p_{\lambda} p^{\sigma} f_{\chi}^{(0)} \delta^{\prime}\left(p^{2}\right)
\end{aligned}
$$

and using the Schouten identity

$$
p_{\mu} \epsilon_{\nu \rho \sigma \lambda}+p_{\nu} \epsilon_{\rho \sigma \lambda \mu}+p_{\rho} \epsilon_{\sigma \lambda \mu \nu}+p_{\sigma} \epsilon_{\lambda \mu \nu \rho}+p_{\lambda} \epsilon_{\mu \nu \rho \sigma}=0,
$$


we obtain

$$
\begin{aligned}
\epsilon_{\mu \nu \rho \sigma} F^{\rho \lambda} p_{\lambda} p^{\sigma} f_{\chi}^{(0)} \delta^{\prime}\left(p^{2}\right) & =p_{\mu} \tilde{F}_{\nu \sigma} p^{\sigma} f_{\chi}^{(0)} \delta^{\prime}\left(p^{2}\right)-p_{\nu} \tilde{F}_{\mu \sigma} p^{\sigma} f_{\chi}^{(0)} \delta^{\prime}\left(p^{2}\right)+p^{2} \tilde{F}_{\mu \nu} f_{\chi}^{(0)} \delta^{\prime}\left(p^{2}\right), \\
\epsilon_{\mu \nu \rho \sigma} \nabla^{\rho} \mathcal{F}_{\chi}^{(0) \sigma} & =-2 Q \tilde{F}_{\mu \nu} f_{\chi}^{(0)} \delta\left(p^{2}\right)+\epsilon_{\mu \nu \rho \sigma} p^{\sigma}\left(\nabla^{\rho} f_{\chi}^{(0)}\right) \delta\left(p^{2}\right)-2 Q \epsilon_{\mu \nu \rho \sigma} F^{\rho \lambda} p_{\lambda} p^{\sigma} f_{\chi}^{(0)} \delta^{\prime}\left(p^{2}\right), \\
& =\epsilon_{\mu \nu \rho \sigma} p^{\sigma}\left(\nabla^{\rho} f_{\chi}^{(0)}\right) \delta\left(p^{2}\right)-2 Q\left(p_{\mu} \tilde{F}_{\nu \sigma}-p_{\nu} \tilde{F}_{\mu \sigma}\right) p^{\sigma} f_{\chi}^{(0)} \delta^{\prime}\left(p^{2}\right) .
\end{aligned}
$$

Here, $\delta^{\prime}\left(p^{2}\right)=d \delta\left(p^{2}\right) / d p^{2}$, and we have used the relation $p^{2} \delta^{\prime}\left(p^{2}\right)=-\delta\left(p^{2}\right)$. Now, Eq. (48) becomes

$$
\epsilon_{\mu \nu \rho \sigma} p^{\sigma}\left(\nabla^{\rho} f_{\chi}^{(0)}\right) \delta\left(p^{2}\right)-2 Q\left(p_{\mu} \tilde{F}_{\nu \sigma}-p_{\nu} \tilde{F}_{\mu \sigma}\right) p^{\sigma} f_{\chi}^{(0)} \delta^{\prime}\left(p^{2}\right)=-2 \chi\left(p_{\mu} \mathcal{F}_{\nu, \chi}^{(1)}-p_{\nu} \mathscr{F}_{\mu, \chi}^{(1)}\right) .
$$

Contracting both sides of the above equation with $p^{\nu}$ and using Eq. (49), one can derive that

$$
p^{2}\left(Q \tilde{F}_{\mu \sigma} p^{\sigma} f_{\chi}^{(0)} \delta^{\prime}\left(p^{2}\right)-\chi \mathcal{F}_{\mu, \chi}^{(1)}\right)=0
$$

hence, the most general solution to the above constraint equation can be written as

$$
\mathscr{F}_{\mu, \chi}^{(1)}=\mathscr{H}_{\mu} \delta\left(p^{2}\right)+\chi Q \tilde{F}_{\mu \nu} p^{\nu} f_{\chi}^{(0)} \delta^{\prime}\left(p^{2}\right) .
$$

In the above, the $\mathscr{H}_{\mu}$ is an arbitrary Lorentz vector. By using the second constraint (49), one arrives at

$$
p^{\mu} \mathscr{H}_{\mu} \delta\left(p^{2}\right)=0
$$

Because of the special nature of lightlike momentum $p^{\mu}=$ $(|\mathbf{p}|, \mathbf{p})$ (as mandated by the delta function), there are three categories of vectors that can satisfy the above equation: one parallel to $p_{\mu}$ itself and the other two taking the form $(0, \mathbf{K})$ with the spatial component satisfying $\mathbf{K} \cdot \mathbf{p}=0$. Thus, one can decompose $\mathscr{H}_{\mu}$ into components that are parallel/orthogonal to the momentum $p^{\mu}$ respectively:

$$
\mathscr{H}_{\mu}=p_{\mu} f_{\chi}^{(1)}+\mathscr{K}_{\mu} .
$$

Here, $f_{\chi}^{(1)}$ has the natural interpretation as the first-order correction to $f_{\chi}^{(0)}$ by comparing the above with the zerothorder Eq. (45). To solve the orthogonal part $\mathscr{K}^{\mu}$, one can substitute the representation of $\mathscr{F}_{\mu, \chi}^{(1)}$ into Eq. (52) and get the following constraint equation for $\mathscr{K}^{\mu}$ :

$$
\epsilon_{\mu \nu \rho \sigma} p^{\sigma}\left(\nabla^{\rho} f_{\chi}^{(0)}\right) \delta\left(p^{2}\right)=-2 \chi\left(p_{\mu} \mathscr{K}_{\nu}-p_{\nu} \mathscr{K}_{\mu}\right) \delta\left(p^{2}\right) .
$$

The most general solution of $\mathscr{K}_{\mu}$ can be expressed as

$$
\mathscr{K}_{\mu}=\frac{\chi}{2 p \cdot n} \epsilon_{\mu \nu \lambda \rho} p^{\nu} n^{\lambda}\left(\nabla^{\rho} f_{\chi}^{(0)}\right),
$$

where an arbitrary auxiliary timelike unit vector $n^{\mu}$ (satisfying $n^{\mu} n_{\mu}=1$ ) has been introduced. It should be noted that the above is the correct solution to the constraint equations even for space time-dependent $n^{\mu}(x)$. A detailed proof of this solution is included in Appendix. The meaning of $n^{\mu}$ and the pertinent frame-dependence issue will be discussed in the next subsection.

Finally, we can combine the solutions to the zeroth- and first-order constraint equations and write down the following expression of $\mathscr{F}_{\chi}^{\mu}$ up to the first order of $\hbar$ :

$$
\begin{aligned}
\mathcal{F}_{\chi}^{\mu}= & p^{\mu} f_{\chi} \delta\left(p^{2}\right)+\hbar \chi Q \tilde{F}^{\mu \nu} p_{\nu} f_{\chi}^{(0)} \delta^{\prime}\left(p^{2}\right) \\
& -\hbar \frac{\chi}{2 p \cdot n} \epsilon^{\mu \nu \lambda \rho} n_{\nu} p_{\lambda}\left(\nabla_{\rho} f_{\chi}^{(0)}\right) \delta\left(p^{2}\right) .
\end{aligned}
$$

Here, $\tilde{F}^{\mu \nu}=\frac{1}{2} \epsilon_{\mu \nu \rho \sigma} F^{\rho \sigma}$ is the dual tensor of $F^{\mu \nu}$. We have introduced the distribution function $f_{\chi}$ including $\hbar$-order quantum correction,

$$
f_{\chi}=f_{\chi}^{(0)}+\hbar f_{\chi}^{(1)}
$$

which can also be decomposed into positive/negative energy parts, like Eq. (45), $f_{\chi}(x, p)=\sum_{\epsilon= \pm 1} \theta\left(\epsilon p^{0}\right) f_{\chi}^{\epsilon} \times$ $(x, \epsilon p)$. Now, the chiral current is given by

$$
J_{\chi}^{\mu}=\int d^{4} p \mathscr{J}_{\chi}^{\mu}=J_{\chi}^{(0) \mu}+\hbar J_{\chi}^{(1) \mu},
$$

with the zeroth-order $J_{\chi}^{(0) \mu}$ and first-order $J_{\chi}^{(1) \mu}$ expressed as

$$
\begin{aligned}
J_{\chi}^{(0) \mu}= & \int d^{4} p p^{\mu} f_{\chi}^{(0)} \delta\left(p^{2}\right), \\
J_{\chi}^{(1) \mu}= & \int d^{4} p p^{\mu} f_{\chi}^{(1)} \delta\left(p^{2}\right)+\chi Q \tilde{F}^{\mu \nu} \int d^{4} p p_{\nu} f_{\chi}^{(0)} \delta^{\prime}\left(p^{2}\right) \\
& -\frac{\chi}{2} \epsilon^{\mu \nu \lambda \rho} n_{\nu} \int d^{4} p \frac{1}{p \cdot n} p_{\lambda}\left(\nabla_{\rho} f_{\chi}^{(0)}\right) \delta\left(p^{2}\right) .
\end{aligned}
$$

Similarly, one can also get the expression of the vector/axial currents and energy-momentum tensor from $\mathcal{F}_{\chi}^{\mu}$. 


\section{Lorentz invariance and frame dependence}

In the solution for $\mathscr{K}^{\mu}$ in Eq. (58), an arbitrary auxiliary quantity $n^{\mu}$ appears to be a free choice at our disposal without clear physical meaning. A more careful examination reveals that the quantity $n^{\mu}$ actually plays a subtle yet crucial role in the chiral transport, especially pertaining to the confusing issues of Lorentz invariance and frame dependence, which we shall discuss next.

To understand the role of $n^{\mu}$, let us come back to the decomposition of $\mathscr{H}_{\mu}$ in Eq. (56), i.e., $\mathscr{H}_{\mu}=p_{\mu} f_{\chi}^{(1)}+\mathcal{K}_{\mu}$. As already mentioned above and as discussed with great detail in Appendix, this decomposition is subtle due to the lightlike nature of the $p^{\mu}$. To unambiguously identify the first-order correction to the distribution function, one must demand that the part along $p_{\mu}$ should be attributed to the distribution term $f_{\chi}^{(1)}$ while the rest should be attributed to the $\mathscr{K}_{\mu}$ term. In fact, such a requirement completely fixes the form of $\mathscr{K}_{\mu}$. For a uniquely defined $f_{\chi}^{(1)}$, the $\mathscr{K}_{\mu}$ must take the form $(0, \mathbf{K})$ with the spatial component satisfying $\mathbf{K} \cdot \mathbf{p}=0$. Combining this requirement with Eq. (58), one arrives at the unique choice $n^{\mu}=(1,0,0,0)$ and the corresponding $\mathscr{K}_{\mu}$ below:

$$
\mathscr{K}_{\mu}=\left(0, \frac{\chi}{2|\mathbf{p}|} \mathbf{p} \times\left(\vec{\nabla} f_{\chi}^{(0)}\right)\right) .
$$

This, however, is not the end of the story. While the above construction gives well-defined $f_{\chi}^{(1)} p_{\mu}$ and $\mathscr{K}_{\mu}$ in the current reference frame, this decomposition is actually frame dependent. To appreciate this less obvious subtlety, suppose in the current frame there is a vector $\mathscr{K}_{\mu}=(0, \mathbf{K})$ that satisfies orthogonality to $p_{\mu}$ via $\mathbf{K} \cdot \mathbf{p}=0$. But upon boosting into a different frame with both $p_{\mu}$ and $\mathscr{K}_{\mu}$ transformed as Lorentz vectors into $p^{\prime}$ and $\mathscr{K}^{\prime}$, one finds that in general $\mathscr{K}^{\prime}$ acquires a component along $p^{\prime}$, despite the fact that they still satisfy $\mathscr{K}^{\prime} \cdot p^{\prime}=0$. That means one has to redo the proper decomposition in the new reference frame and find a different $\mathscr{K}^{\prime \prime}=\left(0, \mathbf{K}^{\prime \prime}\right)$ satisfying $\mathbf{K}^{\prime \prime} \cdot p^{\prime}=0$. This issue again arises from the lightlike nature of $p_{\mu}$

A lengthy calculation in Appendix proves that if one boosts from the current frame to a different frame of 4velocity $u^{\mu}$ (with respect to the current frame) then the $\mathscr{K}_{\mu}$ from proper decomposition in this new frame should be precisely and uniquely given by Eq. (58) with the identification $n^{\mu} \rightarrow u^{\mu}$, which leaves a well-defined $f_{\chi}^{(1)}$ in this new reference frame. Hence, the role of $n^{\mu}$ now becomes clear. This result also explicates the fact that the distribution term $f_{\chi}^{(1)}$ becomes frame dependent as well. While the distribution function in usual transport theory is a Lorentz scalar, here it is demonstrated clearly that in chiral transport theory a nontrivial frame dependence of the distribution function arises precisely at the $\hat{\mathbf{O}}(\hbar)$-order correction and in the specific way discussed above.

In short, the Wigner function formalism is in itself totally covariant, and it is the decomposition of $\mathscr{H}_{\mu}$ that introduces frame dependence. The unique identification of $f_{\chi}^{(1)}$ requires the $\mathscr{K}^{\mu}$ to contain no $p^{\mu}$-parallel component while this requirement is frame dependent. For an observer with velocity $u^{\mu}=n^{\mu}$, Eq. (58) gives the correct $\mathscr{K}^{\mu}$. The peculiar structure of $\mathscr{K}^{\mu}$ also clarifies the frame dependence of spin tensor $S^{\mu \nu}$ and the side-jump effect $[30,35,36]$.

\section{COVARIANT CHIRAL TRANSPORT EQUATION}

\section{A. Covariant chiral transport equation}

In this subsection, we focus on deriving the covariant chiral transport equation up to $\hbar$ order, which can be obtained by substituting Eq. (59) into Eq. (35):

$$
\begin{aligned}
0 & =\nabla_{\mu} \mathcal{F}_{\chi}^{\mu} \\
& =\nabla_{\mu}\left(p^{\mu} f_{\chi} \delta\left(p^{2}\right)\right)+\hbar \chi Q \nabla_{\mu}\left(\tilde{F}^{\mu \nu} f_{\chi}^{(0)} p_{\nu} \delta^{\prime}\left(p^{2}\right)\right)-\hbar \frac{\chi}{2} \epsilon^{\mu \nu \lambda \rho} \nabla_{\mu}\left(\frac{1}{p \cdot n} n_{\nu} p_{\lambda}\left(\nabla_{\rho} f_{\chi}^{(0)}\right) \delta\left(p^{2}\right)\right)
\end{aligned}
$$

One can further simplify the first term of the above equation as

$$
\nabla_{\mu}\left(p^{\mu} f_{\chi} \delta\left(p^{2}\right)\right)=\delta\left(p^{2}\right) p \cdot \nabla f_{\chi}
$$

the second term as

$$
\begin{aligned}
\nabla_{\mu}\left(\tilde{F}^{\mu \nu} f_{\chi}^{(0)} p_{\nu} \delta^{\prime}\left(p^{2}\right)\right) & =-Q \tilde{F}^{\mu \nu} F_{\mu \nu} f_{\chi}^{(0)} \delta^{\prime}\left(p^{2}\right)-2 Q \tilde{F}^{\mu \nu} p_{\nu} F_{\mu \lambda} p^{\lambda} f_{\chi}^{(0)} \delta^{\prime \prime}\left(p^{2}\right)+\tilde{F}^{\mu \nu} p_{\nu}\left(\nabla_{\mu} f_{\chi}^{(0)}\right) \delta^{\prime}\left(p^{2}\right) \\
& =-Q \tilde{F}^{\mu \nu} F_{\mu \nu} f_{\chi}^{(0)} \delta^{\prime}\left(p^{2}\right)-\frac{1}{2} Q \tilde{F}^{\mu \nu} F_{\mu \nu} p^{2} f_{\chi}^{(0)} \delta^{\prime \prime}\left(p^{2}\right)+\tilde{F}^{\mu \nu} p_{\nu}\left(\nabla_{\mu} f_{\chi}^{(0)}\right) \delta^{\prime}\left(p^{2}\right) \\
& =\tilde{F}^{\mu \nu} p_{\nu}\left(\nabla_{\mu} f_{\chi}^{(0)}\right) \delta^{\prime}\left(p^{2}\right)
\end{aligned}
$$


and the third term as

$$
\begin{aligned}
\epsilon^{\mu \nu \lambda \rho} \nabla_{\mu}\left(\frac{1}{p \cdot n} n_{\nu} p_{\lambda}\left(\nabla_{\rho} f_{\chi}^{(0)}\right) \delta\left(p^{2}\right)\right)= & 2 Q \tilde{F}^{\rho \lambda} p_{\lambda}\left(\nabla_{\rho} f_{\chi}^{(0)}\right) \delta^{\prime}\left(p^{2}\right)+\frac{2 Q}{p \cdot n} p_{\lambda} \tilde{F}^{\lambda \nu} n_{\nu} \delta^{\prime}\left(p^{2}\right) p \cdot \nabla f_{\chi}^{(0)} \\
& -\frac{1}{(p \cdot n)^{2}}\left[\left(\partial_{\mu} n_{\sigma}\right) p^{\sigma}-Q F_{\mu \alpha} n^{\alpha}\right] \epsilon^{\mu \nu \lambda \rho} n_{\nu} p_{\lambda}\left(\nabla_{\rho} f_{\chi}^{(0)}\right) \delta\left(p^{2}\right) \\
& +\frac{1}{p \cdot n} \epsilon^{\mu \nu \lambda \rho}\left(\partial_{\mu} n_{\nu}\right) p_{\lambda}\left(\nabla_{\rho} f_{\chi}^{(0)}\right) \delta\left(p^{2}\right)-\frac{Q}{p \cdot n} p_{\lambda}\left(\partial_{\sigma} \tilde{F}^{\lambda \nu}\right) n_{\nu}\left(\partial_{p}^{\sigma} f_{\chi}^{(0)}\right) \delta\left(p^{2}\right) .
\end{aligned}
$$

In the above steps, we have used the relation $p^{2} \delta^{\prime}\left(p^{2}\right)=-\delta\left(p^{2}\right), p^{2} \delta^{\prime \prime}\left(p^{2}\right)=-2 \delta^{\prime}\left(p^{2}\right)$, the Bianchi identity $\nabla_{\mu} \tilde{F}^{\mu \nu}=\partial_{\mu} \tilde{F}^{\mu \nu}=0$, and $4 \tilde{F}^{\mu \nu} p_{\nu} F_{\mu \alpha} p^{\alpha}=p^{2} \tilde{F}^{\mu \nu} F_{\mu \nu}$, which can be easily proved by the Schouten identity (51). Also, we have used the following relations:

$$
\begin{aligned}
\epsilon^{\mu \nu \lambda \rho}\left(\nabla_{\mu} \nabla_{\rho} f_{\chi}^{(0)}\right) & =\frac{1}{2} \epsilon^{\mu \nu \lambda \rho}\left[\nabla_{\mu}, \nabla_{\rho}\right] f_{\chi}^{(0)}=Q\left(\partial_{\sigma} \tilde{F}^{\nu \lambda}\right) \partial_{p}^{\sigma} f_{\chi}^{(0)} \\
2 p^{\alpha} \epsilon^{\mu \nu \lambda \rho} n_{\nu} p_{\lambda} F_{\mu \alpha} & =-2(p \cdot n) \tilde{F}^{\rho \lambda} p_{\lambda}+2 p^{2} \tilde{F}^{\rho \nu} n_{\nu}-2 p_{\lambda} \tilde{F}^{\lambda \nu} n_{\nu} p_{\rho} .
\end{aligned}
$$

Finally, we obtain the following covariant chiral kinetic equation as the evolution equation for the distribution function $f_{\chi}$ up to $\hbar$-order quantum correction:

$$
\begin{aligned}
0=\nabla_{\mu} \mathcal{F}^{\mu}= & \delta\left(p^{2}\right) p \cdot \nabla f_{\chi}-\hbar \frac{\chi Q}{p \cdot n} p_{\lambda} \tilde{F}^{\lambda \nu} n_{\nu} \delta^{\prime}\left(p^{2}\right) p \cdot \nabla f_{\chi}^{(0)} \\
& +\hbar \frac{\chi}{2(p \cdot n)^{2}}\left[\left(\partial_{\mu} n_{\sigma}\right) p^{\sigma}-Q F_{\mu \alpha} n^{\alpha}\right] \epsilon^{\mu \nu \lambda \rho} n_{\nu} p_{\lambda}\left(\nabla_{\rho} f_{\chi}^{(0)}\right) \delta\left(p^{2}\right) \\
& -\hbar \frac{\chi}{2 p \cdot n} \epsilon^{\mu \nu \lambda \rho}\left(\partial_{\mu} n_{\nu}\right) p_{\lambda}\left(\nabla_{\rho} f_{\chi}^{(0)}\right) \delta\left(p^{2}\right)+\hbar \frac{\chi Q}{2 p \cdot n} p_{\lambda}\left(\partial_{\sigma} \tilde{F}^{\lambda \nu}\right) n_{\nu}\left(\partial_{p}^{\sigma} f_{\chi}^{(0)}\right) \delta\left(p^{2}\right) \\
= & \delta\left(p^{2}-\hbar \frac{\chi Q}{p \cdot n} p_{\lambda} \tilde{F}^{\lambda \nu} n_{\nu}\right)\left\{p \cdot \nabla+\hbar \frac{\chi}{2(p \cdot n)^{2}}\left[\left(\partial_{\mu} n_{\sigma}\right) p^{\sigma}-Q F_{\mu \alpha} n^{\alpha}\right] \epsilon^{\mu \nu \lambda \rho} n_{\nu} p_{\lambda} \nabla_{\rho}\right. \\
& \left.-\hbar \frac{\chi}{2 p \cdot n} \epsilon^{\mu \nu \lambda \rho}\left(\partial_{\mu} n_{\nu}\right) p_{\lambda} \nabla_{\rho}+\hbar \frac{\chi Q}{2 p \cdot n} p_{\lambda}\left(\partial_{\sigma} \tilde{F}^{\lambda \nu}\right) n_{\nu} \partial_{p}^{\sigma}\right\} f_{\chi} .
\end{aligned}
$$

In the last step, we used the Taylor expansion in the $\delta$ function, and we only keep terms up to the $\hbar$ order. One can see from the argument of the delta function that the energy of the chiral particle has been shifted in $\hbar$ order, showing the effect of quantum correction. Equation (64) is the complete and consistent covariant chiral kinetic equation. Notably, the mass shell condition in the delta function has shifted from the classical case and receives an $\hbar$-order quantum correction that has the physical interpretation of magnetization energy due to interaction between the charged chiral fermion's magnetic moment with the external magnetic field. Again, it is worth emphasizing that the expression of distribution function $f_{\chi}$, or more strictly speaking the firstorder correction $f_{\chi}^{(1)}$, depends on the choice of $n^{\mu}$.

\section{B. 3D chiral kinetic equation}

In this subsection, let us consider a simplified case and take $n^{\mu}$ as a constant-homogeneous 4 -vector $u^{\mu}$. In this case, Eq. (64) can be written as

$$
0=\delta\left(p^{2}-\hbar \frac{\chi Q}{p \cdot u}(B \cdot p)\right)\left\{p^{\rho} \nabla_{\rho}-\hbar \frac{\chi Q}{2(p \cdot u)^{2}} \epsilon^{\mu \nu \lambda \rho} E_{\mu} u_{\nu} p_{\lambda} \nabla_{\rho}+\hbar \frac{\chi Q}{2 p \cdot u} p_{\lambda}\left(\partial_{\rho} B^{\lambda}\right) \partial_{p}^{\rho}\right\} f_{\chi}
$$

where we introduce the notations $E^{\mu}=F^{\mu \nu} u_{\nu}, B^{\mu}=\tilde{F}^{\mu \nu} u_{\nu}$. In addition, using the relations

$$
\begin{aligned}
F^{\mu \nu} & =E^{\mu} u^{\nu}-E^{\nu} u^{\mu}+\epsilon^{\mu \nu \rho \sigma} u_{\rho} B_{\sigma}, \\
\epsilon^{\mu \nu \lambda \rho} \epsilon_{\rho \alpha \beta \delta} & =\delta_{\alpha}^{\mu} \delta_{\beta}^{\nu} \delta_{\delta}^{\lambda}+\delta_{\beta}^{\mu} \delta_{\delta}^{\nu} \delta_{\alpha}^{\lambda}+\delta_{\delta}^{\mu} \delta_{\alpha}^{\nu} \delta_{\beta}^{\lambda}-\delta_{\alpha}^{\nu} \delta_{\beta}^{\mu} \delta_{\delta}^{\lambda}-\delta_{\beta}^{\nu} \delta_{\delta}^{\mu} \delta_{\alpha}^{\lambda}-\delta_{\delta}^{\nu} \delta_{\alpha}^{\mu} \delta_{\beta}^{\lambda},
\end{aligned}
$$


Eq. (65) can be reduced to

$$
\begin{aligned}
0= & \delta\left(p^{2}-\hbar \frac{\chi Q}{p \cdot u}(B \cdot p)\right)\left\{\left(p^{\rho}-\hbar \frac{\chi Q}{2(p \cdot u)^{2}} \epsilon^{\mu \nu \lambda \rho} E_{\mu} u_{\nu} p_{\lambda}\right) \partial_{\rho}\right. \\
& +Q\left[-(E \cdot p) u^{\rho}+(p \cdot u) E^{\rho}+\epsilon^{\mu \nu \rho \sigma} p_{\mu} u_{\nu} B_{\sigma}\right. \\
& \left.\left.+\hbar \frac{\chi Q}{2(p \cdot u)^{2}}\left((B \cdot p) E^{\rho}-(E \cdot B) \bar{p}^{\rho}\right)+\hbar \frac{\chi}{2 p \cdot u} p_{\lambda}\left(\partial^{\rho} B^{\lambda}\right)\right] \partial_{\rho}^{p}\right\} f_{\chi} .
\end{aligned}
$$

One could further simplify the above equation by choosing $n^{\mu}=u^{\mu}=(1,0,0,0)$, which can be achieved by a proper Lorentz transformation. In this frame, $E^{\mu}=(0, \mathbf{E}), B^{\mu}=(0, \mathbf{B}), \bar{p}^{\rho}=(0, \mathbf{p})$ is the 3-momentum, $p \cdot u=p_{0}$ is the energy, $B \cdot p=-\mathbf{B} \cdot \mathbf{p}, E \cdot p=-\mathbf{E} \cdot \mathbf{p}$, and $E \cdot B=-\mathbf{E} \cdot \mathbf{B}$. From the delta function of Eq. (66), we can get the shifted energy in the external field up to $\hbar$ order,

$$
p_{0}=\epsilon|\mathbf{p}|\left(1-\hbar \epsilon Q \mathbf{B} \cdot \mathbf{b}_{\chi}\right)=\epsilon E_{\mathbf{p}}, \quad E_{\mathbf{p}}=|\mathbf{p}|\left(1-\hbar \epsilon Q \mathbf{B} \cdot \mathbf{b}_{\chi}\right),
$$

where $\mathbf{b}_{\chi}=\chi \frac{\mathbf{p}}{2|\mathbf{p}|^{3}}$ is the Berry curvature, $\hat{\mathbf{p}}=\mathbf{p} /|\mathbf{p}|$ is the unit vector of the momentum, and $\epsilon= \pm 1$ correspond to the particle with positive/negative energy. With the shifted energy, the group velocity of the quasiparticle becomes

$$
\tilde{\mathbf{v}}=\frac{\partial E_{\mathbf{p}}}{\partial \mathbf{p}}=\hat{\mathbf{p}}\left(1+2 \hbar \epsilon Q \mathbf{B} \cdot \mathbf{b}_{\chi}\right)-\hbar \epsilon Q b_{\chi} \mathbf{B}
$$

Note that the on-shell condition (67) constrains the energy in Eq. (66); hence, it is no longer a free variable in the distribution function. By integrating Eq. (66) over $p_{0}$, one arrives at the following three-dimensional chiral kinetic equation:

$$
\begin{aligned}
\sum_{\epsilon= \pm 1} & \frac{\epsilon E_{\mathbf{p}}}{2}\left\{\frac{1}{E_{\mathbf{p}}} \partial_{t}+\epsilon\left(\frac{p^{k}}{E_{\mathbf{p}}^{2}}+\hbar \frac{\chi Q}{2 E_{\mathbf{p}}^{4}} \epsilon^{i j k} E_{i} p_{j}\right) \partial_{k}\right. \\
& +Q\left[\frac{E^{k}}{E_{\mathbf{p}}}+\epsilon \epsilon^{i j k} \frac{p_{i}}{E_{\mathbf{p}}^{2}} B_{j}-\hbar \epsilon \frac{\chi Q}{2 E_{\mathbf{p}}^{4}}\left((\mathbf{B} \cdot \mathbf{p}) E^{k}-(\mathbf{E} \cdot \mathbf{B}) p^{k}\right)-\hbar \frac{\chi}{2 E_{\mathbf{p}}^{3}}\left(\partial^{k} \mathbf{B} \cdot \mathbf{p}\right)\right] \partial_{k}^{p} \\
& \left.+Q\left[-E^{k} \frac{p_{k}}{E_{\mathbf{p}}^{2}}-\hbar \epsilon \frac{\chi}{2 E_{\mathbf{p}}^{3}}\left(\partial_{t} \mathbf{B} \cdot \mathbf{p}\right)\right] \partial_{E_{\mathbf{p}}}\right\} f_{\chi}^{\epsilon}\left(x, E_{\mathbf{p}}, \epsilon \mathbf{p}\right)=0 .
\end{aligned}
$$

By expanding various powers of the energy $E_{\mathbf{p}}$ in $\hbar$ and keeping terms up to the first order, one obtains

$$
\begin{aligned}
& \sum_{\epsilon= \pm 1} \frac{\epsilon}{2}\left(1-\hbar \epsilon Q \mathbf{B} \cdot \mathbf{b}_{\chi}\right)\left\{\left(1+\hbar \epsilon Q \mathbf{B} \cdot \mathbf{b}_{\chi}\right) \partial_{t}+\epsilon\left(\hat{p}^{k}\left(1+2 \hbar \epsilon Q \mathbf{B} \cdot \mathbf{b}_{\chi}\right)+\hbar Q \epsilon^{i j k} E_{i} b_{\chi j}\right) \partial_{k}\right. \\
& \quad+Q\left[E^{k}+\frac{1}{\epsilon Q}\left(\partial^{k} E_{\mathbf{p}}\right)+\epsilon \epsilon^{i j k} \hat{p}_{i} B_{j}\left(1+2 \hbar \epsilon Q \mathbf{B} \cdot \mathbf{b}_{\chi}\right)+\hbar \epsilon Q(\mathbf{E} \cdot \mathbf{B}) b_{\chi}^{k}\right] \partial_{k}^{p} \\
& \left.\quad+\left[-Q E^{k} \cdot \hat{p}_{k}\left(1+2 \hbar \epsilon Q \mathbf{B} \cdot \mathbf{b}_{\chi}\right)+\left(\partial_{t} E_{\mathbf{p}}\right)\right] \partial_{E_{\mathbf{p}}}\right\} f_{\chi}^{\epsilon}\left(x, E_{\mathbf{p}}, \epsilon \mathbf{p}\right)=0 .
\end{aligned}
$$

The next step is to turn the energy-derivative terms into the derivative terms with respect to the actual independent variables (i.e., space-time coordinates and 3-momentum):

$$
\begin{aligned}
& \sum_{\epsilon= \pm 1} \frac{\epsilon}{2}\left(1-\hbar \epsilon Q \mathbf{B} \cdot \mathbf{b}_{\chi}\right)\left\{\left(1+\hbar \epsilon Q \mathbf{B} \cdot \mathbf{b}_{\chi}\right)\left(\partial_{t}+\left(\partial_{t} E_{\mathbf{p}}\right) \partial_{E_{\mathbf{p}}}\right)\right. \\
& \quad+\epsilon\left(\hat{p}^{k}\left(1+2 \hbar \epsilon Q \mathbf{B} \cdot \mathbf{b}_{\chi}\right)+\hbar Q \epsilon^{i j k} E_{i} b_{\chi j}\right)\left(\partial_{k}+\left(\partial_{k} E_{\mathbf{p}}\right) \partial_{E_{\mathbf{p}}}\right) \\
& \left.\quad+Q\left[E^{k}+\frac{1}{\epsilon Q}\left(\partial^{k} E_{\mathbf{p}}\right)+\epsilon \epsilon^{i j k} \hat{p}_{i} B_{j}\left(1+2 \hbar \epsilon Q \mathbf{B} \cdot \mathbf{b}_{\chi}\right)-\hbar \epsilon Q(\mathbf{E} \cdot \mathbf{B}) b_{\chi}^{k}\right]\left(\partial_{k}^{p}+\left(\partial_{k}^{p} E_{\mathbf{p}}\right) \partial_{E_{\mathbf{p}}}\right)\right\} f_{\chi}^{\epsilon}\left(x, E_{\mathbf{p}}, \epsilon \mathbf{p}\right)=0 .
\end{aligned}
$$


Using the expression $\tilde{v}_{k}=-\partial_{k}^{p} E_{\mathbf{p}}$, Eq. (71) can be further simplified,

$$
\begin{aligned}
& \sum_{\epsilon= \pm 1} \frac{\epsilon}{2}\left(1-\hbar \epsilon Q \mathbf{B} \cdot \mathbf{b}_{\chi}\right)\left\{\left(1+\hbar \epsilon Q \mathbf{B} \cdot \mathbf{b}_{\chi}\right)\left(\partial_{t}+\left(\partial_{t} E_{\mathbf{p}}\right) \partial_{E_{\mathbf{p}}}\right)\right. \\
& \quad+\epsilon\left(\tilde{v}^{k}+\hbar \epsilon Q\left(\tilde{\mathbf{v}} \cdot \mathbf{b}_{\chi}\right) B^{k}+\hbar Q \epsilon^{i j k} E_{i} b_{\chi j}\right)\left(\partial_{k}+\left(\partial_{k} E_{\mathbf{p}}\right) \partial_{E_{\mathbf{p}}}\right) \\
& \left.\quad+Q\left[E^{k}+\frac{1}{\epsilon Q}\left(\partial^{k} E_{\mathbf{p}}\right)+\epsilon \epsilon^{i j k} \tilde{v}_{i} B_{j}+\hbar \epsilon Q(\mathbf{E} \cdot \mathbf{B}) b_{\chi}^{k}\right]\left(\partial_{k}^{p}+\left(\partial_{k}^{p} E_{\mathbf{p}}\right) \partial_{E_{\mathbf{p}}}\right)\right\} f_{\chi}^{\epsilon}\left(x, E_{\mathbf{p}}, \epsilon \mathbf{p}\right)=0 .
\end{aligned}
$$

It can be seen explicitly that by employing the chain rule

$$
\begin{aligned}
{\left[\partial_{t}+\left(\partial_{t} E_{\mathbf{p}}\right) \partial_{E_{\mathbf{p}}}\right] f_{\chi}^{\epsilon}\left(t, \mathbf{x}, E_{\mathbf{p}}, \epsilon \mathbf{p}\right) } & =\partial_{t} f_{\chi}^{\epsilon}(t, \mathbf{x}, \epsilon \mathbf{p}), \\
{\left[\partial_{k}+\left(\partial_{k} E_{\mathbf{p}}\right) \partial_{E_{\mathbf{p}}}\right] f_{\chi}^{\epsilon}\left(t, \mathbf{x}, E_{\mathbf{p}}, \epsilon \mathbf{p}\right) } & =\partial_{k} f_{\chi}^{\epsilon}(t, \mathbf{x}, \epsilon \mathbf{p}), \\
{\left[\partial_{k}^{p}+\left(\partial_{k}^{p} E_{\mathbf{p}}\right) \partial_{E_{\mathbf{p}}}\right] f_{\chi}^{\epsilon}\left(t, \mathbf{x}, E_{\mathbf{p}}, \epsilon \mathbf{p}\right) } & =\partial_{k}^{p} f_{\chi}^{\epsilon}(t, \mathbf{x}, \epsilon \mathbf{p})
\end{aligned}
$$

one can eventually remove the energy derivative terms and obtain

$$
\begin{aligned}
& \sum_{\epsilon= \pm 1} \epsilon\left\{\partial_{t}+\epsilon\left(1-\hbar \epsilon Q \mathbf{B} \cdot \mathbf{b}_{\chi}\right)\left(\tilde{v}^{k}+\hbar \epsilon Q\left(\tilde{\mathbf{v}} \cdot \mathbf{b}_{\chi}\right) B^{k}+\hbar Q \epsilon^{i j k} E_{i} b_{\chi j}\right) \partial_{k}\right. \\
& \left.\quad+Q\left(1-\hbar \epsilon Q \mathbf{B} \cdot \mathbf{b}_{\chi}\right)\left[E^{k}+\frac{1}{\epsilon Q}\left(\partial^{k} E_{\mathbf{p}}\right)+\epsilon \epsilon^{i j k} \tilde{v}_{i} B_{j}+\hbar \epsilon Q(\mathbf{E} \cdot \mathbf{B}) b_{\chi}^{k}\right] \partial_{k}^{p}\right\} f_{\chi}^{\epsilon}(t, \mathbf{x}, \epsilon \mathbf{p})=0,
\end{aligned}
$$

Contracting over all of index $i, j, k=1,2,3$ and replacing $\mathbf{p}$ by $\epsilon \mathbf{p}$ to convert distribution of a particle with negative energy into that of an antiparticle, we can write the chiral kinetic equation for particle and antiparticle separately,

$$
\left\{\partial_{t}+\frac{1}{\sqrt{G}}\left(\tilde{\mathbf{v}}+\hbar Q\left(\tilde{\mathbf{v}} \cdot \mathbf{b}_{\chi}\right) \mathbf{B}+\hbar Q \tilde{\mathbf{E}} \times \mathbf{b}_{\chi}\right) \cdot \nabla_{\mathbf{x}}+\frac{\epsilon Q}{\sqrt{G}}\left(\tilde{\mathbf{E}}+\tilde{\mathbf{v}} \times \mathbf{B}+\hbar Q(\tilde{\mathbf{E}} \cdot \mathbf{B}) \mathbf{b}_{\chi}\right) \cdot \nabla_{\mathbf{p}}\right\} f_{\chi}^{\epsilon}(x, \mathbf{p})=0 .
$$

Here, $\sqrt{G}=\left(1+\hbar Q \mathbf{b}_{\chi} \cdot \mathbf{B}\right)$ corresponds to the Jacobian, and

$$
\tilde{\mathbf{E}}=\mathbf{E}-\frac{1}{\epsilon Q} \nabla_{\mathbf{x}} E_{\mathbf{p}}, \quad E_{\mathbf{p}}=|\mathbf{p}|\left(1-\hbar Q \mathbf{B} \cdot \mathbf{b}_{\chi}\right), \quad \tilde{\mathbf{v}}=\frac{\partial E_{\mathbf{p}}}{\partial \mathbf{p}}=\hat{\mathbf{p}}\left(1+2 \hbar Q \mathbf{B} \cdot \mathbf{b}_{\chi}\right)-\hbar Q b_{\chi} \mathbf{B},
$$

where the $\chi$ denotes the chiral or the helicity and $f_{\chi}^{\epsilon}$ indicates the distribution function of a given chiral particle or antiparticle. One can also convert Eq. (74) into the equation for particles with particular helicity $h \equiv \epsilon \chi$,

$$
\left\{\partial_{t}+\frac{1}{\sqrt{G}}\left(\tilde{\mathbf{v}}+\hbar \epsilon Q\left(\tilde{\mathbf{v}} \cdot \mathbf{b}_{h}\right) \mathbf{B}+\hbar \epsilon Q \tilde{\mathbf{E}} \times \mathbf{b}_{h}\right) \cdot \nabla_{\mathbf{x}}+\frac{\epsilon Q}{\sqrt{G}}\left(\tilde{\mathbf{E}}+\tilde{\mathbf{v}} \times \mathbf{B}+\hbar \epsilon Q(\tilde{\mathbf{E}} \cdot \mathbf{B}) \mathbf{b}_{h}\right) \cdot \nabla_{\mathbf{p}}\right\} f_{h}^{\epsilon}(x, \mathbf{p})=0 .
$$

This reproduces the well-known three-dimensional chiral kinetic equation $[29,35,36]$, with the corresponding Jacobian, energy, group velocity given by

$$
\begin{aligned}
& \sqrt{G}=\left(1+\hbar \epsilon Q \mathbf{b}_{h} \cdot \mathbf{B}\right), \quad \tilde{\mathbf{E}}=\mathbf{E}-\frac{1}{\epsilon Q} \nabla_{\mathbf{x}} E_{\mathbf{p}}, \\
& E_{\mathbf{p}}=|\mathbf{p}|\left(1-\hbar \epsilon Q \mathbf{B} \cdot \mathbf{b}_{h}\right), \quad \tilde{\mathbf{v}}=\frac{\partial E_{\mathbf{p}}}{\partial \mathbf{p}}=\hat{\mathbf{p}}\left(1+2 \hbar \epsilon Q \mathbf{B} \cdot \mathbf{b}_{h}\right)-\hbar \epsilon Q b_{h} \mathbf{B} .
\end{aligned}
$$

Therefore, the chiral kinetic equation (75) is derived from a complete and consistent analysis of the Wigner function formalism with the semiclassical expansion method.

\section{CONCLUSION}

In this paper, we have derived a covariant and complete solution (59) for the chiral component of the Wigner function, along with the corresponding chiral transport equation (64) for massless Dirac fermions, by starting from the general Wigner function formalism and carrying out a consistent semiclassical expansion up to $\hat{\mathbf{O}}(\hbar)$ order. A detailed proof is given for the general and unique solution of the peculiar component $\mathscr{K}_{\mu}$ in the $\hat{\mathbf{O}}(\hbar)$-order chiral component of the Wigner function. In particular, this new analysis clarifies exactly why and how the 
Lorentz-invariance and frame-dependence issues associated with the $\hat{\mathbf{O}}(\hbar)$ correction to the phase space distribution function arise within a totally covariant framework. From the so-obtained chiral transport equation, one also naturally derives as its consequences the $3 \mathrm{D}$ formulation of chiral kinetic theory as well as many special features of chiral fermions such as the magnetization energy shift, the Berry curvature, chiral anomaly, CME, etc. The covariant chiral transport theory lays a firm conceptual foundation for describing anomalous transport in the generally nonequilibrium systems of chiral fermions.

We end by discussing a number of extensions and applications within the current framework. First of all, it is of great interest to explore higher-order quantum effects beyond just the $\hat{\mathbf{O}}(\hbar)$ order, and in this regard, the Wigner function formalism has its unique advantage. Second, it is also highly interesting to develop the equal-time quantum transport theory [53] for chiral fermions in this framework. The 3D chiral kinetic theory only preserves the zeroth moment information of the four-dimensional theory, and there is a whole hierarchy of equations for higher moments of the four-dimensional theory that together forms the equal-time transport theory, which turns four-dimensional theory into a complete initial problem and is crucial for phenomenological applications. Furthermore, while we focus on the vector and axial components of the Wigner function in this paper, the other components also bear nontrivial physical meanings for physically relevant quantities such as spin density and helicity density, which could be readily studied with the same approach as in this paper [60]. Additionally, in the current formalism, it is relatively straightforward to incorporate fermion collision terms by starting from a Dirac Lagrangian including interaction terms [51,52], which is also important for phenomenology. Last but not least, the role of a small nonzero mass (and generally the quantum transport of massive fermions) could be easily explored in the Wigner function formalism along lines similar to the present study. These problems will be investigated in the future.

\section{ACKNOWLEDGMENTS}

The authors thank Jianhua Gao, Xingyu Guo, Xu-Guang Huang, Shi Pu, and Qun Wang for helpful discussions. The research of A. H. and P.Z. is supported by the NSFC and MOST Grants No. 11335005, No. 11575093, No. 2013CB922000, and No. 2014CB845400. J. L. acknowledges support by the NSFC Grant No. 11735007. J.L. and S. S. are supported in part by the National Science Foundation under Grant No. PHY1352368 and by the U.S. Department of Energy, Office of Science, Office of Nuclear Physics, within the framework of the Beam Energy Scan Theory Topical Collaboration. Y. J. is supported by the startup funding of Beihang University.

\section{APPENDIX: DISCUSSIONS ON $\mathcal{K}^{\mu}$}

In this Appendix, we discuss the solution of $\mathscr{K}^{\mu}$ [Eq. (58)] in Sec. III A. To obtain the first-order correction of the chiral vector, we need to solve the vector $\mathscr{K}^{\mu}$ satisfying Eq. (57),

$\epsilon_{\mu \nu \rho \sigma} p^{\sigma}\left(\nabla^{\rho} f_{\chi}^{(0)}\right) \delta\left(p^{2}\right)=-2 \chi\left(p_{\mu} \mathscr{K}_{\nu}-p_{\nu} \mathscr{K}_{\mu}\right) \delta\left(p^{2}\right)$.

Let us denote $A^{\rho} \equiv \frac{1}{2} \chi\left(\nabla^{\rho} f_{\chi}^{(0)}\right) \delta\left(p^{2}\right)$ and $K^{\mu} \equiv \mathscr{K}^{\mu} \delta\left(p^{2}\right)$; the equation becomes

$$
\epsilon_{\mu \nu \rho \sigma} p^{\sigma} A^{\rho}=-\left(p_{\mu} K_{\nu}-p_{\nu} K_{\mu}\right)
$$

Noting that the Vlasov equation as in Eq. (47),

$$
\delta\left(p^{2}\right) p^{\mu} \nabla_{\mu} f_{\chi}^{(0)}=0
$$

requires $p_{\mu} A^{\mu}=0$, one can derive that

$$
p_{\mu} K^{\mu}=0, \quad A_{\mu} K^{\mu}=0 .
$$

This indicates that the unknown $K^{\mu}$ vector is orthogonal to two known vectors $A^{\mu}$ and $p^{\mu}$ orthogonal to each other, the latter of which is a null vector. In principle, in the $3+1$ dimensional space-time, there should be unique solution of $K^{\mu}$, with an undetermined component parallel to $p^{\mu}$.

To see this, let us first consider a simplified case: if taking the null vector $p^{\mu}=(E, E, 0,0)$, then one could always write down its orthogonal vectors as

$$
A^{\mu}=(a, a, b, c), \quad K^{\mu}=(k, k, d, f),
$$

and $A, K$ 's being orthogonal yields $b d+c f=0$, which is similar to the two-dimensional orthogonal condition. Substituting this in Eq. (A2), one could find

$$
d=-c, \quad f=b .
$$

This indicates that for any given known $p^{\mu}$ and $A^{\rho} \equiv$ $\frac{1}{2} \chi\left(\nabla^{\rho} f_{\chi}^{(0)}\right) \delta\left(p^{2}\right)$ we can fix $K^{\mu}$ except its component parallel to $p^{\mu}$. As a matter of fact, such a conclusion is valid not only in the frame in which $p^{\mu}=(E, E, 0,0)$ but also in any general case. Being any null vector, $p^{\mu}$ can always be expressed by its direction angle $\theta$ and $\phi$,

$$
p^{\mu}=E(1, \sin \theta \cos \phi, \sin \theta \sin \phi, \cos \theta)
$$

hence, its two orthogonal vectors can be expanded in the corresponding basis: 


$$
\begin{aligned}
& A^{\mu}=a(1, \sin \theta \cos \phi, \sin \theta \sin \phi, \cos \theta)+b(0, \cos \theta \cos \phi, \cos \theta \sin \phi,-\sin \theta)+c(0,-\sin \phi, \cos \phi, 0), \\
& K^{\mu}=k(1, \sin \theta \cos \phi, \sin \theta \sin \phi, \cos \theta)+d(0, \cos \theta \cos \phi, \cos \theta \sin \phi,-\sin \theta)+f(0,-\sin \phi, \cos \phi, 0) .
\end{aligned}
$$

To solve $K^{\mu}$, we introduced an arbitrary auxiliary timelike vector $n^{\mu}=\left(n^{t}, n^{x}, n^{y}, n^{z}\right)$, normalized to unity, $n^{\mu} n_{\mu}=1$, and constructed the solution as in Eq. (58),

$$
K^{\mu}=\frac{\epsilon^{\mu \nu \rho \sigma} p_{\nu} n_{\rho} A_{\sigma}}{n \cdot p} .
$$

First of all, let us show that Eq. (A6) gives a valid solution to Eqs. (57) and (A2). Substituting the solution in the right-hand side of Eq. (A2), one obtains

$$
\begin{aligned}
-\left(p_{\mu} K_{\nu}-p_{\nu} K_{\mu}\right) & =\frac{p_{\nu} \epsilon_{\mu \alpha \rho \sigma} p^{\alpha} n^{\rho} A^{\sigma}}{n \cdot p}-\frac{p_{\mu} \epsilon_{\nu \alpha \rho \sigma} p^{\alpha} n^{\rho} A^{\sigma}}{n \cdot p} \\
& =\frac{p_{\nu} \epsilon_{\mu \alpha \rho \sigma} p^{\alpha} n^{\rho} A^{\sigma}}{n \cdot p}+\frac{p_{\nu} \epsilon_{\alpha \rho \sigma \mu} p^{\alpha} n^{\rho} A^{\sigma}}{n \cdot p}+\frac{p_{\alpha} \epsilon_{\rho \sigma \mu \nu} p^{\alpha} n^{\rho} A^{\sigma}}{n \cdot p}+\frac{p_{\rho} \epsilon_{\sigma \mu \nu \alpha} p^{\alpha} n^{\rho} A^{\sigma}}{n \cdot p}+\frac{p_{\sigma} \epsilon_{\mu \nu \alpha \rho} p^{\alpha} n^{\rho} A^{\sigma}}{n \cdot p} \\
& =\frac{p_{\nu} \epsilon_{\mu \alpha \rho \sigma} p^{\alpha} n^{\rho} A^{\sigma}}{n \cdot p}-\frac{p_{\nu} \epsilon_{\mu \alpha \rho \sigma} p^{\alpha} n^{\rho} A^{\sigma}}{n \cdot p}+\frac{(p \cdot p) \epsilon_{\rho \sigma \mu \nu} n^{\rho} A^{\sigma}}{n \cdot p}+\frac{(n \cdot p) \epsilon_{\sigma \mu \nu \alpha} p^{\alpha} A^{\sigma}}{n \cdot p}+0 \\
& =\epsilon_{\mu \nu \rho \sigma} p^{\sigma} A^{\rho},
\end{aligned}
$$

which satisfies the equality.

Second, after some tedious but straightforward steps, one can compute the coefficients in Eq. (A5) as

$$
\begin{aligned}
& d=-c, \\
& f=b, \\
& k=\frac{b\left(-n^{x} \sin \phi+n^{y} \cos \phi\right)-c\left(n^{x} \cos \theta \cos \phi+n^{y} \cos \theta \sin \phi-n^{z} \sin \theta\right)}{p \cdot n} .
\end{aligned}
$$

We can see explicitly that no matter what $n^{\mu}$ field we choose it gives the same component of $K^{\mu}$ orthogonal to the momentum $p^{\mu}$. It shows that Eq. (58) gives a valid and complete solution of $\mathscr{K}^{\mu}$, as long as we constrain $n^{\mu}$ to be timelike, which ensures $p \cdot n \neq 0$.

On the other hand, as can be seen in Eq. (A13), different $n^{\mu}$ influence the component parallel to $p^{\mu}$. To understand the role of $n^{\mu}$ and why it may cause ambiguity in $\mathscr{K}^{\mu}$, let us carefully consider the decomposition $\mathscr{H}^{\mu} \equiv p^{\mu} f_{\chi}^{(1)}+\mathscr{K}^{\mu}$, trying to separate the vector $\mathscr{C}^{\mu}$ orthogonal to $p^{\mu}$ into two parts. This decomposition is, however, subtle due to the lightlike nature of $p, p^{\mu} p_{\mu}=0$; i.e., $p$ is "self-orthogonal." It is worth commenting that this lightlike feature is of course ultimately because the chiral fermion is massless. To avoid ambiguity of the decomposition, one can always ensure that $f_{\chi}^{(1)}$ contains all $p^{\mu}$-parallel components by constraining

$$
p^{0} \mathscr{K}^{0}-\sum_{i=1}^{3} p^{i} \mathscr{K}^{i}=0, \quad p^{0} \mathscr{K}^{0}+\sum_{i=1}^{3} p^{i} \mathscr{K}^{i}=0,
$$

or equivalently

$$
p \cdot \mathscr{K}=0, \quad \mathscr{K}^{0}=0
$$

Such a requirement can be achieved by taking $n^{\mu}=$ $(1,0,0,0)$, which yields $k=0$ in Eq. (A13), and

$$
\begin{aligned}
\overline{\mathscr{K}}^{\mu} & =\left.\frac{\epsilon^{\mu \nu \rho \sigma} p_{\nu} n_{\rho}\left(\nabla_{\sigma} f_{\chi}^{(0)}\right)}{2 \chi(n \cdot p)}\right|_{n=(1,0,0,0)} \\
& =\left(0,-\frac{\chi}{2|\mathbf{p}|} \mathbf{p} \times\left(\boldsymbol{\nabla} f_{\chi}^{(0)}\right)\right) .
\end{aligned}
$$


For an observer in the lab frame, Eq. (A15) gives the complete decomposition of $\mathscr{H}^{\mu}$. However, this is not the end of the story-such a characteristic is not boost invariant, due to the fact that the requirement of "orthogonality" is not Lorentz invariant. One can find a vector $\mathscr{K}^{\mu}$ orthogonal to a null vector $p^{\mu}$ by restricting $\mathscr{K}^{0}=0, p \cdot \mathscr{K}=0$, but it is impossible to maintain $\mathscr{K}^{\prime 0}=$ 0 under arbitrary Lorentz transformation $\mathscr{K}^{\prime \mu}=\Lambda_{\nu}^{\mu} \mathscr{K}^{\nu}$, $p^{\prime \mu}=\Lambda_{\nu}^{\mu} p^{\nu}$. To see this explicitly, for an observer moving with velocity $u^{\mu}$, the transformation $\Lambda_{\nu}^{\mu}$ is given by the element in the $(\mu+1)$ th row, $(\nu+1)$ th column of the matrix,

$$
\left(\begin{array}{cccc}
u^{t} & -u^{x} & -u^{y} & -u^{z} \\
-u^{x} & 1+\frac{u^{x} u^{x}}{1+u^{t}} & \frac{u^{x} u^{y}}{1+u^{t}} & \frac{u^{x} u^{z}}{1+u^{t}} \\
-u^{y} & \frac{u^{y} u^{x}}{1+u^{t}} & 1+\frac{u^{y} u^{y}}{1+u^{t}} & \frac{u^{y} u^{z}}{1+u^{t}} \\
-u^{z} & \frac{u^{z} u^{x}}{1+u^{t}} & \frac{u^{z} u^{y}}{1+u^{t}} & 1+\frac{u^{z} u^{z}}{1+u^{t}}
\end{array}\right)
$$

while in his local rest frame,

$$
\begin{aligned}
\overline{\mathscr{K}}^{\prime 0} & =\Lambda_{\nu}^{0} \overline{\mathscr{K}}^{\nu}=\frac{\chi}{2|\mathbf{p}|} \mathbf{u} \cdot \mathbf{p} \times\left(\boldsymbol{\nabla} f_{\chi}^{(0)}\right) \\
& =\frac{\chi}{2|\mathbf{p}|}(\mathbf{u} \times \mathbf{p}) \cdot\left(\boldsymbol{\nabla} f_{\chi}^{(0)}\right) \neq 0 .
\end{aligned}
$$

Hence, the decomposition of $\mathscr{H}^{\mu}$ is frame dependent, and one should determine $f_{\chi}^{(1)}$ and $\mathscr{K}^{\mu}$ differently, with respect to different frame. As a matter of fact, for the observer moving with velocity $u^{\mu}$, one can construct $\mathscr{K}^{\mu}$ as

$$
\mathscr{K}^{\mu}=\frac{\chi}{2 u \cdot p} \epsilon^{\mu \nu \rho \sigma} p_{\nu} u_{\rho}\left(\nabla_{\sigma} f_{\chi}^{(0)}\right),
$$

where the time component of vector $\mathscr{K}$ vanishes in his local rest frame:

$$
\begin{aligned}
\mathscr{K}^{\prime 0} & =\Lambda_{\nu}^{0} \mathscr{K}^{\nu}=u_{\nu}\left(\frac{\chi}{2 u \cdot p} \epsilon^{\nu \mu \rho \sigma} p_{\mu} u_{\rho}\left(\nabla_{\sigma} f_{\chi}^{(0)}\right)\right) \\
& =\frac{\chi}{2 u \cdot p} \epsilon^{\nu \mu \rho \sigma} p_{\mu} u_{\nu} u_{\rho}\left(\nabla_{\sigma} f_{\chi}^{(0)}\right)=0 .
\end{aligned}
$$

Actually, it is more obvious if one expresses all quantities in the observer's local rest frame:

$$
\begin{aligned}
\mathscr{K}^{\prime \mu} & =\Lambda_{\nu}^{\mu} \frac{\chi}{2 u \cdot p} \epsilon^{\nu \lambda \rho \sigma} p_{\lambda} u_{\rho}\left(\nabla_{\sigma} f_{\chi}^{(0)}\right) \\
& =\frac{\chi}{2 u^{\prime} \cdot p^{\prime}} \Lambda_{\nu}^{\mu} \Lambda_{\alpha}^{\nu} \Lambda_{\beta}^{\lambda} \Lambda_{\kappa}^{\rho} \Lambda_{\delta}^{\sigma} \epsilon^{\alpha \beta \kappa \delta} p_{\lambda} u_{\rho}\left(\nabla_{\sigma} f_{\chi}^{(0)}\right) \\
& =\frac{\chi}{2 u^{\prime} \cdot p^{\prime}} \epsilon^{\mu \beta \kappa \delta} p_{\beta}^{\prime} u_{\kappa}^{\prime}\left(\nabla_{\delta}^{\prime} f_{\chi}^{(0)}\right) \\
& =\left.\frac{\chi}{2 u^{\prime} \cdot p^{\prime}} \epsilon^{\mu \nu \rho \sigma} p_{\nu}^{\prime} u_{\rho}^{\prime}\left(\nabla_{\sigma}^{\prime} f_{\chi}^{(0)}\right)\right|_{u^{\prime}=(1,0,0,0)} \\
& =\left(0,-\frac{\chi}{2\left|\mathbf{p}^{\prime}\right|} \mathbf{p}^{\prime} \times\left(\nabla^{\prime} f_{\chi}^{(0)}\right)\right) .
\end{aligned}
$$

Consequently, one can see that constructing $\mathscr{K}_{\mu}$ as in Eq. (58) with arbitrary timelike vector $n^{\mu}$ has the following physical meaning: for an observer moving with velocity $u^{\mu}=n^{\mu}, \mathscr{K}^{\mu} \equiv \frac{\epsilon^{\mu \nu \rho \sigma} p_{\nu} n_{\rho}\left(\nabla_{\sigma} f_{\chi}^{(0)}\right)}{2 \chi(n \cdot p)}$ contains no $p^{\mu}$-parallel component in his local rest frame. It gives a complete decomposition of $\mathscr{H}^{\mu}$, and $f_{\chi}^{(1)}$ corresponds to the first-order correction of the distribution function observed in this frame. This reflects the frame dependence of spin tensor $S^{\mu \nu} \equiv \lambda \frac{\epsilon^{\mu \nu \rho \sigma} p_{\rho} n_{\sigma}}{p \cdot n}$ as mentioned in Refs. [29,35,36].

It is worth mentioning that $\mathscr{K}^{\mu}$ in Eq. (58) is a vector defined in the lab frame, and once $n$ is fixed, it transforms like a Lorentz vector under boost transformation. It has the meaning of what is known by an observer in the lab frame about the proper decomposition for another observer moving with velocity $n$. As illustrated in Eqs. (A11)-(A13), the $\mathscr{K}^{\mu}$ vectors, corresponding to observers moving with velocities $u$ and $v$, respectively, differ with a $p^{\mu}$-parallel component:

$$
\begin{aligned}
\mathscr{K}_{[u]}^{\mu}-\mathscr{K}_{[v]}^{\mu}= & \frac{\chi}{2 u \cdot p} \epsilon^{\mu \nu \rho \sigma} p_{\nu} u_{\rho}\left(\nabla_{\sigma} f_{\chi}^{(0)}\right)-\frac{\chi}{2 v \cdot p} \epsilon^{\mu \nu \rho \sigma} p_{\nu} v_{\rho}\left(\nabla_{\sigma} f_{\chi}^{(0)}\right) \\
= & \frac{\chi}{2(u \cdot p)(v \cdot p)}\left[v_{\alpha} p^{\alpha} \epsilon^{\mu \nu \rho \sigma} p_{\nu} u_{\rho}\left(\nabla_{\sigma} f_{\chi}^{(0)}\right)-u_{\alpha} p^{\alpha} \epsilon^{\mu \nu \rho \sigma} p_{\nu} v_{\rho}\left(\nabla_{\sigma} f_{\chi}^{(0)}\right)\right] \\
= & \frac{\chi}{2(u \cdot p)(v \cdot p)}\left[v_{\alpha} p^{\alpha} \epsilon^{\mu \nu \rho \sigma} p_{\nu} u_{\rho}\left(\nabla_{\sigma} f_{\chi}^{(0)}\right)+u_{\alpha} p^{\mu} \epsilon^{\nu \rho \sigma \alpha} p_{\nu} v_{\rho}\left(\nabla_{\sigma} f_{\chi}^{(0)}\right)+u_{\alpha} p^{\nu} \epsilon^{\rho \sigma \alpha \mu} p_{\nu} v_{\rho}\left(\nabla_{\sigma} f_{\chi}^{(0)}\right)\right. \\
& +u_{\alpha} p^{\rho} \epsilon^{\sigma \alpha \mu \nu} p_{\nu} v_{\rho}\left(\nabla_{\sigma} f_{\chi}^{(0)}\right)+u_{\alpha} p^{\sigma} \epsilon^{\alpha \mu \nu \rho} p_{\nu} v_{\rho}\left(\nabla_{\sigma} f_{\chi}^{(0)}\right) \\
= & \frac{\chi}{2(u \cdot p)(v \cdot p)}\left[v_{\alpha} p^{\alpha} \epsilon^{\mu \nu \rho \sigma} p_{\nu} u_{\rho}\left(\nabla_{\sigma} f_{\chi}^{(0)}\right)+p^{\mu} \epsilon^{\nu \alpha \rho \sigma} p_{\nu} u_{\alpha} v_{\rho}\left(\nabla_{\sigma} f_{\chi}^{(0)}\right)+(p \cdot p) \epsilon^{\mu \rho \alpha \sigma} v_{\rho} u_{\alpha}\left(\nabla_{\sigma} f_{\chi}^{(0)}\right)\right. \\
& \left.-v_{\rho} p^{\rho} \epsilon^{\mu \nu \alpha \sigma} p_{\nu} u_{\alpha}\left(\nabla_{\sigma} f_{\chi}^{(0)}\right)+\epsilon^{\mu \nu \alpha \rho} p_{\nu} u_{\alpha} v_{\rho}\left(p \cdot \nabla f_{\chi}^{(0)}\right)\right] \\
= & \frac{\chi \epsilon^{\nu \alpha \rho \sigma} p_{\nu} u_{\alpha} v_{\rho}\left(\nabla_{\sigma} f_{\chi}^{(0)}\right)}{2(u \cdot p)(v \cdot p)} p^{\mu} .
\end{aligned}
$$


Noting that the vector $\mathscr{H}^{\mu}$ should be frame independent,

$$
\mathscr{H}_{[u]}^{\mu}=\mathscr{K}_{[u]}^{\mu}+p^{\mu} f_{[u], \mathcal{X}}^{(1)} \equiv \mathscr{H}_{[v]}^{\mu}=\mathscr{K}_{[v]}^{\mu}+p^{\mu} f_{[v], \mathcal{X}}^{(1)}
$$

one can explicitly see the difference between distributions observed in the $u$ and $v$ frames:

$$
f_{[u], \chi}-f_{[v], \chi}=\hbar\left(f_{[u], \chi}^{(1)}-f_{[v], \chi}^{(1)}\right)=-\frac{\hbar \chi \epsilon^{\nu \alpha \rho \sigma} p_{\nu} u_{\alpha} v_{\rho}\left(\nabla_{\sigma} f_{\chi}^{(0)}\right)}{2(u \cdot p)(v \cdot p)} .
$$

[1] D. Kharzeev, Phys. Lett. B 633, 260 (2006).

[2] D. Kharzeev and A. Zhitnitsky, Nucl. Phys. A797, 67 (2007).

[3] D. E. Kharzeev, L. D. McLerran, and H. J. Warringa, Nucl. Phys. A803, 227 (2008).

[4] K. Fukushima, D. E. Kharzeev, and H. J. Warringa, Phys. Rev. D 78, 074033 (2008).

[5] D. T. Son and P. Surowka, Phys. Rev. Lett. 103, 191601 (2009).

[6] D. E. Kharzeev and D. T. Son, Phys. Rev. Lett. 106, 062301 (2011).

[7] Y. Jiang, X. G. Huang, and J. Liao, Phys. Rev. D 92, 071501 (2015).

[8] J. Erdmenger, M. Haack, M. Kaminski, and A. Yarom, J. High Energy Phys. 01 (2009) 055.

[9] N. Banerjee, J. Bhattacharya, S. Bhattacharyya, S. Dutta, R. Loganayagam, and P. Surowka, J. High Energy Phys. 01 (2011) 094.

[10] M. Torabian and H. U. Yee, J. High Energy Phys. 08 (2009) 020.

[11] T. Kalaydzhyan and I. Kirsch, Phys. Rev. Lett. 106, 211601 (2011).

[12] S. Pu, J. h. Gao, and Q. Wang, Phys. Rev. D 83, 094017 (2011).

[13] D. E. Kharzeev and H. U. Yee, Phys. Rev. D 84, 045025 (2011).

[14] M. A. Metlitski and A. R. Zhitnitsky, Phys. Rev. D 72, 045011 (2005).

[15] G. M. Newman and D. T. Son, Phys. Rev. D 73, 045006 (2006).

[16] J. Charbonneau and A. Zhitnitsky, J. Cosmol. Astropart. Phys. 08 (2010) 010.

[17] M. Lublinsky and I. Zahed, Phys. Lett. B 684, 119 (2010).

[18] M. Asakawa, A. Majumder, and B. Muller, Phys. Rev. C 81, 064912 (2010).

[19] K. Landsteiner, E. Megias, and F. Pena-Benitez, Phys. Rev. Lett. 107, 021601 (2011).

[20] D. E. Kharzeev, J. Liao, S. A. Voloshin, and G. Wang, Prog. Part. Nucl. Phys. 88, 1 (2016).

[21] D. E. Kharzeev, Annu. Rev. Nucl. Part. Sci. 65, 193 (2015).

[22] J. Liao, Pramana 84, 901 (2015).
[23] K. Hattori and X. G. Huang, Nucl. Sci. Tech. 28, 26 (2017).

[24] A. A. Burkov, J. Phys. Condens. Matter 27, 113201 (2015).

[25] E. M. Lifshitz and L. P. Pitaevskii, Physical Kinetics (Pergamon, New York, 1981).

[26] D. T. Son and N. Yamamoto, Phys. Rev. Lett. 109, 181602 (2012).

[27] D. T. Son and N. Yamamoto, Phys. Rev. D 87, 085016 (2013).

[28] M. A. Stephanov and Y. Yin, Phys. Rev. Lett. 109, 162001 (2012).

[29] J. Y. Chen, D. T. Son, M. A. Stephanov, H. U. Yee, and Y. Yin, Phys. Rev. Lett. 113, 182302 (2014).

[30] J. Y. Chen, D. T. Son, and M. A. Stephanov, Phys. Rev. Lett. 115, 021601 (2015).

[31] D. E. Kharzeev, M. A. Stephanov, and H. U. Yee, Phys. Rev. D 95, 051901 (2017).

[32] J. W. Chen, S. Pu, Q. Wang, and X. N. Wang, Phys. Rev. Lett. 110, 262301 (2013).

[33] J. H. Gao, Z.T. Liang, S. Pu, Q. Wang, and X. N. Wang, Phys. Rev. Lett. 109, 232301 (2012).

[34] J. H. Gao, S. Pu, and Q. Wang, Phys. Rev. D 96, 016002 (2017).

[35] Y. Hidaka, S. Pu, and D. L. Yang, Phys. Rev. D 95, 091901 (2017).

[36] Y. Hidaka, S. Pu, and D. L. Yang, Phys. Rev. D 97, 016004 (2018).

[37] N. Mueller and R. Venugopalan, Phys. Rev. D 97, 051901 (2018).

[38] N. Mueller and R. Venugopalan, Phys. Rev. D 96, 016023 (2017).

[39] E. V. Gorbar, V. A. Miransky, I. A. Shovkovy, and P. O. Sukhachov, Phys. Rev. B 95, 205141 (2017).

[40] Y. Wu, D. Hou, and H. c. Ren, Phys. Rev. D 96, 096015 (2017).

[41] M. Mace, S. Schlichting, and R. Venugopalan, Phys. Rev. D 93, 074036 (2016).

[42] M. Mace, N. Mueller, S. Schlichting, and S. Sharma, Phys. Rev. D 95, 036023 (2017).

[43] N. Mller, S. Schlichting, and S. Sharma, Phys. Rev. Lett. 117, 142301 (2016).

[44] K. Fukushima, Phys. Rev. D 92, 054009 (2015). 
[45] S. Ebihara, K. Fukushima, and S. Pu, Phys. Rev. D 96, 016016 (2017).

[46] Y. Sun, C. M. Ko, and F. Li, Phys. Rev. C 94, 045204 (2016).

[47] Y. Sun and C. M. Ko, Phys. Rev. C 95, 034909 (2017).

[48] A. Huang, Y. Jiang, S. Shi, J. Liao, and P. Zhuang, Phys. Lett. B 777, 177 (2018).

[49] Y. Jiang, S. Shi, Y. Yin, and J. Liao, Chin. Phys. C 42, 011001 (2018).

[50] S. Shi, Y. Jiang, E. Lilleskov, and J. Liao, Ann. Phys. (Amsterdam) 394, 50 (2018).

[51] P. Zhuang and U. W. Heinz, Ann. Phys. (N.Y.) 245, 311 (1996).

[52] P. Zhuang and U. W. Heinz, Phys. Rev. D 53, 2096 (1996).

[53] P. Zhuang and U. W. Heinz, Phys. Rev. D 57, 6525 (1998).
[54] S. Ochs and U. W. Heinz, Ann. Phys. (N.Y.) 266, 351 (1998).

[55] D. Vasak, M. Gyulassy, and H. T. Elze, Ann. Phys. (N.Y.) 173, 462 (1987).

[56] X. Guo and P. Zhuang, Phys. Rev. D 98, 016007 (2018).

[57] S. R. De Groot, W. A. Van Leeuwen, and C. G. Van Weert, Relativistic Kinetic Theory. Principles and Applications (North-Holland, Amsterdam, 1980).

[58] I. Bialynicki-Birula, P. Gornicki, and J. Rafelski, Phys. Rev. D 44, 1825 (1991).

[59] K. Yagi, T. Hatsuda, and Y. Miake, Cambridge Monogr. Part. Phys., Nucl. Phys., Cosmol. 23, 1 (2005).

[60] A. Huang et al. (to be published). 